Cahiers Société

\title{
Autopsie d'une erreur de jeunesse : les apories de la critique marxienne de l'institutionnalisme de Hegel
}

\section{Jean-François Filion}

Numéro 2, décembre 2020

Marx, critique du capital et de la société

URI : https://id.erudit.org/iderudit/1075558ar

DOI : https://doi.org/10.7202/1075558ar

Aller au sommaire du numéro

Éditeur(s)

Collectif Société

ISSN

2562-5373 (imprimé)

2562-5381 (numérique)

Découvrir la revue

Citer cet article

Filion, J.-F. (2020). Autopsie d'une erreur de jeunesse : les apories de la critique marxienne de l'institutionnalisme de Hegel. Cahiers Société, (2), 171-203.

https://doi.org/10.7202/1075558ar
Résumé de l'article

La Contribution à la critique de la philosophie du droit de Hegel, rédigée en 1843 par un Karl Marx alors âgé de vingt-cinq ans, s'avère une oeuvre cruciale de l'histoire de la pensée, qui demeure aujourd'hui d'une grande importance théorique et pratique. C'est en ayant à l'esprit le fait que Marx adhère à une forme d'optimisme anthropologique méconnaissant la nécessité de la médiation politico-juridique pour l'autonomie subjective que nous tenterons d'exposer en quoi son rejet du soi-disant " mysticisme " hégélien s'avère une brillante erreur de jeunesse qu'il reproduira, cependant, dans ses textes ultérieurs. En effet, après plus d'un siècle de sciences sociales et psychologiques, nous savons que la constitution de l'identité implique une dialectique de la reconnaissance intersubjective garantie par la médiation d'un tiers symbolique institué. Nous proposons également de relativiser la radicalité de la critique marxienne de Hegel en tentant de réinscrire la pensée spéculative des institutions dans la signification ontologique du surgissement de la vie sur Terre et du rôle que prennent les médiations culturelles et politico-juridiques dans le maintien et le développement de l'existence humaine. 


\title{
Autopsie d'une erreur de jeunesse : les apories de la critique marxienne de l'institutionnalisme de Hegel
}

\author{
Jean-François FILION \\ Université du Québec à Montréal
}

La Contribution à la critique de la philosophie du droit de Hegel, titre donné aux manuscrits rédigés par Karl Marx en 1843 à Kreuznach, s'avère une œuvre cruciale de l'histoire de la pensée, qui demeure aujourd'hui d'une grande importance théorique et pratique $^{1}$. Dans le présent article, il ne s'agira pas d'ajouter une nouvelle voix à la Rezeptionsgeschichte de la lecture des Principes de la philosophie du droit par le jeune MarX $^{2}$, mais plutôt d'esquisser une critique de ce qui nous apparaît être une défaillance théorique du marxisme justifiant, depuis Marx lui-même, le rejet unilatéral de la pensée institutionnaliste, laquelle conçoit la structure des médiations politico-juridiques

\footnotetext{
${ }^{1}$ Karl Marx, Contribution à la critique de la philosophie du droit de Hegel, trad. V. Béguin et al., Paris, Éditions sociales, 2018 ; de ces manuscrits publiés par David Riazanov en 1927, il faut en distinguer l'introduction - premier texte de Marx qui mentionne le prolétariat-publiée en 1844 à Paris dans les Annales franco-allemandes (Karl Marx, Contribution à la critique de la philosophie du droit de Hegel. Introduction, dans ibid., p. 285-297). La nouvelle traduction de 2018 améliore grandement la compréhension du commentaire de Marx, notamment parce qu'elle se réfère " en premier lieu » à l'excellente traduction des Principes de la philosophie du droit de J.-F. Kervégan (Paris, Presses Universitaires de France, 2013); néanmoins nous déplorons que les artisans de cette traduction des manuscrits de Kreuznach aient succombé à l'envie de faire preuve d'originalité en traduisant Aufhebung par « relève » (avions-nous besoin d'une énième version - des plus malavisées de surcroît - de ce concept central de l'hégélianisme ?). Par ailleurs, si nous ne recommandons pas les traductions de K. Papaioannou (Critique du droit politique hégélien, Paris, Allia, 2010); de M. Rubel (Critique de la philosophie politique de Hegel [1843] dans Euvres, t. 3 : Philosophie, Paris, Gallimard, coll. « La Pléiade », 1982, p. 863-1018) et de A. Baraquin (Critique du droit politique hégélien, Paris, Éditions sociales, 1975), c'est surtout parce que les extraits de Hegel commentés par Marx y sont mal traduits au point d'en rendre la lecture incompréhensible, par exemple, lorsque « unvernünftigen Wirklichkeit » devient « réalité déraisonnable » plutôt qu' « effectivité irrationnelle ».

${ }^{2}$ Entre autres, Solange Mercier-Josa, Pour lire Hegel et Marx, Paris, Éditions sociales, 1980 ; Retour sur le jeune Marx. Deux études sur le rapport de Marx à Hegel, Paris, Méridiens Klincksieck, 1986 ; Entre Hegel et Marx : Points cruciaux de la philosophie hégélienne du droit, Paris, L'Harmattan, 1999 ; Miguel Abensour, La Démocratie contre l'État. Marx et le moment machiavélien, Paris, Vélin, 2012 ; Marx démocrate. Le Manuscrit de 1843, dir. Étienne Balibar et Gérard Raulet, Paris, Presses Universitaires de France, 2001 ; Kostas Papaioannou, Hegel et Marx : l'Interminable débat, Paris, Allia, 1999; Franck Fischbach, Philosophie de Marx, Paris, Vrin, 2015, p. 73-91 ; Victor Béguin et al., " Introduction », dans Karl Marx, Contribution à la critique de la philosophie du droit de Hegel, op. cit., p. 7-68; et JeanFrançois Kervégan, « Postface. La politique après l'État et la philosophie » dans ibid., p. 331-341.
} 
comme condition ontologique au développement de la liberté individuelle et des activités culturelles, sociales et économiques. Selon le paradigme institutionnaliste, l'individu n'est pas a priori naturellement libre, comme le pose la métaphysique libérale ; l'individu est plutôt préalablement soumis à une solidarité primaire, clanique, patriarcale ou familiale, comme l'ont démontré à plusieurs reprises depuis leur fondation les sciences sociales. Ainsi, les sociétés traditionnelles privilégient la cohésion communautaire au détriment de l'autonomie individuelle, et l'ouverture d'un espace normatif, la société civile, qui offre la possibilité à l'individu d'échapper à l'emprise de la solidarité primaire préexistante, résulte d'une dialectique suivant laquelle les institutions modernes, en premier lieu l'État, vont configurer le droit en fonction d'une métaphysique naturalisant les idéaux de la raison, de la justice et de la liberté. La dimension sociologique propre au paradigme institutionnaliste apparaît dans la reconnaissance du rôle concret que jouent les médiations politico-juridiques dans le processus moderne d'émancipation individuelle, alors que demeure menaçante la présence d'une normativité traditionnelle opposée aux idéaux des Lumières. L'institutionnalisme évalue le fonctionnement des institutions à l'aune de forces politiques concrètes, historiquement présentes, plutôt qu'en rapport à des modèles abstraits et utopiques. Ainsi, la construction politico-juridique de l'autonomie et de la sociabilité, qui ne sont pas innées chez l'humain, implique la médiation d'autorités exerçant une domination instituée au nom de représentations collectives légitimes, et ce, malgré tous les dommages collatéraux de corruption et de discrimination que peut causer une telle dialectique du réel. Les théories sociologiques de Durkheim et de Freitag ainsi que la philosophie hégélienne du droit appartiennent, chacune à sa manière, au paradigme institutionnaliste, qui s'oppose à la fois au traditionalisme conservateur et au progressisme libéral.

Notre hypothèse de lecture consiste à admettre que la Contribution à la critique de la philosophie du droit de Hegel du jeune Marx s'inscrit de plain-pied dans l'antiinstitutionnalisme propre au progressisme libéral, dont l'insuffisance théorique est d'occulter la nécessité de la verticalité institutionnelle pour l'existence de l'autonomie et de la sociabilité humaine. Le présent article vise ainsi à réfuter la critique qu'adresse Marx aux Principes de la philosophie du droit, en dénonçant que la « seule détermination philosophique que Hegel donne du pouvoir gouvernemental est celle de la "subsomption" du singulier et du particulier sous l'universel3 », c'est-à-dire que la domination instituée 4 n'aurait pour effet que d'entraver l'autonomie individuelle.

3 Karl Marx, Contribution à la critique de la philosophie du droit de Hegel, op. cit., p. 138.

4 Michel Freitag conçoit la domination instituée (il dira plutôt institutionnalisée) comme la fixation d'un rapport de force engendré par un état d'exception antéjuridique propre à l'épisode historique de fondation d'une institution politique assumant la stabilisation et la reproduction d'une société. L'institution du pouvoir «implique la capacité de produire ou de s'emparer des moyens de répression, c'est-à-dire d'application de la sanction ». Dans la domination, « l'existence d'un rapport d'asymétrie entre dominants et dominés [...] comporte en même temps sa propre stabilité [...] et un certain degré d'autonomie des agents ou acteurs sociaux qu'il met en présence ». À l'instar de Weber, Freitag considère que la 
Ce rejet radical de la philosophie hégélienne traduit, selon nous, une méconnaissance de la relative fragilité du montage politico-institutionnel qui rend possible l'autonomie individuelle, tout en contribuant au projet révolutionnaire d'une sortie du politique, que Marx précisera après 1843.

Considérer le verdict prononcé par Marx à l'encontre du «mysticisme » de l' « idée politique » hégélienne, en tant que glorification de «l'état de choses existant5 », comme une affaire classée, occulte le fait que la critique marxienne des Principes de la philosophie du droit est tributaire d'une foi inquestionnée en la capacité humaine à la désaliénation intégrale, qui permettrait de faire société sous forme d'" association des producteurs libres ». Le défaut rédhibitoire de ce démocratisme réside dans l'immixtion irréfléchie d'une conception idéaliste de l'être humain qui s'imagine l'horizon d'une multitude de volontés a priori libres parvenant à construire de manière associative, contractuelle, des liens sociaux sans la médiation d'un tiers symbolique institué comportant une monopolisation légitime de la violence. Si nous pouvons comprendre que le jeune Marx de 1843 ait souscrit à un tel projet nourri par un optimisme anthropologique typiquement moderne, nous constatons, après plus d'un siècle de sciences sociales et psychologiques, que la constitution de l'identité implique une dialectique de la reconnaissance intersubjective garantie par la médiation d'un tiers symbolique instituéb. À l'instar des Principes de la philosophie du droit, publiés en 1820, plusieurs théories sociologiques et psychanalytiques ont établi que le développement historique de l'autonomie individuelle a pour condition de possibilité celle du cadre institutionnel où vit l'individu, et ce, en dépit des effets collatéraux, souvent délétères, de la hiérarchie sociale que produit l'existence d'un tel cadre. Cette dialectique permet de concevoir que l'autonomie découle de l'hétéronomie7, c'est-à-dire que la constitution de la réflexivité individuelle implique l'intériorisation d'une normativité donnée a priori par la culture et les institutions qui s'imposent à l'individu à travers son éducation morale avant même qu'il soit en mesure de choisir, ou pas, les normes constitutives de son identité. De ce point de vue, c'est au contraire la dissolution des médiations politico-juridiques, que favorise l'optimisme anthropologique

domination implique absolument la légitimité afin de susciter son acceptation par les dominés (Michel Freitag, Dialectique et Société, vol. 2 : Culture, pouvoir et contrôle : les modes de reproduction formels de la société, Montréal, Saint-Martin et Lausanne, L'Âge d'Homme, 1986, p. 172-173 et 219).

5 Karl Marx, "Postface à la deuxième édition allemande [1873] », Le Capital, livre 1: Le procès de production du capital, trad. J.-P. Lefebvre, Paris, Presses Universitaires de France, 1993, p. 18.

6 Voir Michel Fretiag, "L'identité, l'altérité et le politique », L'oubli de la société. Pour une théorie critique de la postmodernité, Québec/Rennes, Presses de l'Université Laval/Presses Universitaires de Rennes, 2002, p. 183-248. Aussi, dans sa théorie de l'esprit subjectif, Hegel élabore les déterminations intersubjectives de l'identité qui rejettent l'anthropologie abstraite de la modernité ; rappelons enfin l'importance des séminaires de Kojève pour la théorie du stade du miroir chez Lacan.

7 Alain Supiot, Homo juridicus. Essai sur la fonction anthropologique du Droit, Paris, Seuil, 2009, p. 41. 
libéral, qui s'avère une menace pour la liberté8. Ainsi, le paradigme institutionnaliste se résout à reconnaître la nécessité ontologique de positions asymétriques, de rapports de domination et d'interdits indiscutés qui, bien qu'ils puissent produire de manière contingente des effets négatifs (corruptions, oppressions, discriminations, etc.), s'avèrent le prix à payer ${ }^{9}$ afin d'endiguer le déferlement meurtrier que peut engendrer le déni de cette nécessité10.

C'est donc en ayant à l'esprit le fait que Marx adhère à une forme préhégélienne d'optimisme anthropologique ${ }^{11}$ - méconnaissant la nécessité de la médiation politicojuridique, c'est-à-dire de l'esprit objectif, pour l'autonomie subjective - que nous tenterons d'exposer en quoi sa Contribution à la critique de la philosophie du droit de Hegel, écrite à l'âge de 25 ans, s'avère une brillante erreur de jeunesse qu'il reproduira, cependant, dans ses textes ultérieurs. Car, si le vieux Marx considère la Science de la logique comme l'apport majeur de Hegel à la connaissance, il réitèrera jusqu'à la fin son rejet de la philosophie hégélienne du droit parce qu'empreinte de «mysticisme ».

Néanmoins, il est important ici de mentionner que notre critique de l'antiinstitutionnalisme de Marx s'effectue en la séparant préalablement de sa théorie du capital, qui constitue une contribution inestimable à la pensée critique, étant donné que nous distinguons la logique institutionnelle de l'État et la logique organisationnelle du capita112. Cette distinction entre État et capital, ou modernité et capitalisme, s'oppose à leur fusion, qui semble souvent aller de soi dans le marxisme, comme chez Moishe Postone qui, dans sa critique lumineuse de Lukács, fusionne l'esprit objectif conçu par Hegel avec le sujet automate conçu par Marx ${ }^{13}$. Néanmoins, ce choix théorique, motivé

\footnotetext{
8 Sur l'individualisme libéral et ses dangers, voir Michel Freitag, L'abîme de la liberté. Critique du libéralisme, Montréal, Liber, 2012 ; et Dany-Robert Dufour, L'individu qui vient... après le libéralisme, Paris, Denoël, 2011.

9 Prix que peut faire baisser, sans jamais l'éliminer, la vitalité de la disposition d'esprit politique du peuple (voir Jean-François Filion, «Réalisme ontologique et subjectivité politique chez Durkheim : quelques traits hégéliens du fondateur de la sociologie ", Cahiers de recherche sociologique, vol. 56, 2014, p. 109130) et de la common decency, en tant qu'autolimitation de soi de la culture populaire (voir, entre autres, Jean-Claude Michéa, Le Complexe d'Orphée. La gauche, les gens ordinaires et la religion du progrès, Paris, Flammarion, 2014).

10 Voir Jean-Pierre Lebrun, Un monde sans limite, suivi de Malaise dans la subjectivation, Toulouse, Érès, $2^{\mathrm{e}}$ éd., 2016 ; et Pierre Legendre, «La Brèche. Remarques sur la dimension institutionnelle de la Shoah », Sur la question dogmatique en Occident, Paris Fayard, 1999, p. 339-349.

${ }^{11}$ Balibar souligne un retour marxien au Moi fichtéen, lequel illustre l'esprit subjectif typiquement moderne ; voir Étienne Balibar, La philosophie de Marx, Paris, La Découverte, 2001, p. 24-27.

12 Sur la nécessité sociologique de distinguer ces deux logiques, voir dans le présent numéro l'article de François L'Italien, «Phénoménologie de l'esprit pétrifié ». Cette distinction n'est pas nouvelle, car elle correspond analogiquement à celle qu'effectue Weber entre la domination, qui dépend de la capacité à susciter légitimement l'obéissance, et la puissance, qui, tel le capitalisme, s'impose sans devoir obtenir le consentement de ceux qu'elle contraint; voir Max Weber, Concepts fondamentaux de sociologie, trad. J.-P. Grossein, Paris, Gallimard, 2016, p. 159-161.

13 Voir Moishe Postone, « Le sujet de l'histoire : repenser la critique de Hegel dans l'œuvre marxienne de maturité », Actuel Marx, n 50, 2011, p. 61-78. À notre avis, cet amalgame modernité-capitalisme, présent
} 
par la distinction chez Freitag entre le mode de reproduction politico-institutionnel et le mode de reproduction décisionnel-opérationnel, trouve appui au sein du marxisme politique d'Ellen Meiksins Wood, qui distingue avec force les catégories sociologiques de bourgeois et de capitaliste, notamment en insistant sur l'origine agraire de l'accumulation initiale du capital impulsée en Angleterre par les lords, plus féodaux que modernes, et dont la pratique et la Weltanschauung se trouvaient à mille lieues de celles des bourgeois modernes, qui ont renversé en France la monarchie absolutiste ${ }^{14}$. Avec cette distinction, nous considèrerons que Hegel a exposé dans les Principes de la philosophie du droit la quintessence sociopolitique de la modernité bourgeoise précapitaliste. Quelques décennies après la prise de la Bastille, Hegel pouvait encore penser que la modernité allait réaliser une Sittlichkeit, c'est-à-dire une « éthicité » ou une « vie éthique », génératrice de liberté, bien qu'il souligne - insuffisamment il est vrai, et Marx viendra combler le vide - la présence d'une contradiction au cœur du système économique des besoins ${ }^{15}$.

Ainsi, tandis que la conceptualisation de la puissance du capital demeure l'héritage vivant de la pensée de Marx, le présent article traite de ce qui nous y apparaît mort : le rejet unilatéral de l'institutionnalisme. L'idéal d'une conscience de soi a priori sociable, pouvant établir contractuellement des liens sociaux, est trop éloigné de ce que nous a démontré la tradition sociologique, plus proche à cet égard de la Sittlichkeit hégélienne que de la conception associative du lien social proposée par Marx, qui est reprise aujourd'hui dans plusieurs postures critiques. Avec le concept d'éthicité, Hegel dépasse les philosophies politiques modernes, focalisées sur le pouvoir étatique, en y intégrant les sphères de la famille et de la société civile. En concevant une médiation réciproque du politique et du social, Hegel anticipe des aspects fondamentaux des sciences sociales à venir. Or, Marx et la partie la plus radicale de la pensée critique actuelle sombrent dans l'abstraction en proposant une sortie du politique par l'instauration d'une seule organisation sociale dépourvue de verticalité institutionnelle, laquelle est considérée comme l'apanage de « la préhistoire de la société humaine ${ }^{16}$ ». En arrière-plan, le présent article tentera de démontrer que l'anticapitalisme doit s'ouvrir à une éthique de la responsabilité institutionnaliste, en assumant la nécessité

dans des textes théoriques pourtant éclairants à maints égards, handicape grandement la praxis qui se prive ainsi d'une médiation essentielle à la socialité.

14 Ellen Meiksins Wood, L'origine du capitalisme. Une étude approfondie, trad. F. Tétreau, Montréal, Lux, 2009; et George Comninel, Rethinking the French Revolution. Marxism and the revisionist challenge, Londres, Verso, 1991. La distinction entre bourgeois et capitaliste correspond donc sociologiquement à la différence entre logique institutionnelle et logique organisationnelle.

15 G. W. F. Hegel, Principes de la philosophie du droit, trad. J.-F. Kervégan, Paris, Presses Universitaires de France, 2013, § 248, p. 409 ; le capitalisme s'apparente à une «fracture insurmontable » rencontrée par la philosophie hégélienne (Jean-François Kervégan, «L'institution de la liberté », dans ibid., p. 81).

16 Karl Marx, « Préface », dans Contribution à la critique de l'économie politique, Paris, Éditions sociales, 1977, p. 3. 
ontologique de la verticalité instituée, ainsi qu'en rejetant l'imaginaire aussi abstrait que stérile d'un monde idéal sans État, sans travail, sans police, sans argent.

\section{La nature spéculative de l'institutionnalisme hégélien}

Dans les pages qui suivent, nous allons nous aventurer à critiquer ce que Marx considère comme étant problématique dans la philosophie hégélienne du droit qu'il rejette en tant que «mysticisme logique ». Bien qu'il fût l'illustre héritier de Hegel, il nous semble que Marx ait manqué de générosité herméneutique dans son persiflage antispéculatif, qui a marqué durablement la réception négative de l'œuvre hégélienne dans la pensée critique. Si nous pouvons parfois donner raison à Marx lorsqu'il souligne des aspects incongrus dans les Principes de la philosophie du droit, ces incongruités ne suffisent pas à réfuter la démarche hégélienne, d'autant plus que Hegel lui-même, anticipant l'historicité du savoir absolu, se savait « fils de son époque ${ }^{17}$ », donc sujet à des biais risquant d'être ultérieurement dépassés.

Relativiser la portée de la critique marxienne de Hegel en osant donner plus de crédit à la perspective hégélienne peut se faire par l'adoption des précautions suivantes : tout d'abord, il faut appliquer la prudence kantienne minimale qui admet qu'il est tout aussi métaphysique de prouver l'existence d'une entité suprasensible que son inexistence ; ensuite, il faut assumer que l'anthropocentrisme moderne endossé par Marx, mais critiqué par Hegel - qui s'exprime suivant le concept et non du point de vue de l'homme -, est aujourd'hui remis en question par la conscience écologique et l'ouverture à la sagesse orientale; enfin, pour échapper aux soupçons de délire mystique, il faut articuler épistémologiquement une herméneutique faillibiliste à l'ontologie réaliste de l'idéalisme objectif ${ }^{18}$.

\section{Remarques préliminaires}

En 1873, dans la postface à la deuxième édition allemande du Capital, Marx déclare qu'il a « critiqué le côté mystificateur de la dialectique hégélienne il y a près de 30 ans, à une époque où elle était encore à la mode ${ }^{19}$ » et qu'il faut retourner la dialectique, qui est «sur la tête » chez Hegel, "pour découvrir le noyau rationnel sous l'enveloppe mystique 20 ». Bien qu'il se déclare « disciple de ce grand penseur ${ }^{21} »$, la critique véhé-

\footnotetext{
17 G. W. F. Hegel, « Préface », Principes de la philosophie du droit, op. cit., p. 133.

18 Pour s'introduire à ce genre philosophique totalement déphasé avec l'esprit du temps actuel, voir Vittorio Hösle, L'idéalisme objectif, Paris, Cerf, 2001. Par ailleurs, bien que Raymond Ruyer se situe hors de l'hégélianisme, les aspects «panpsychiques » de sa philosophie partagent avec la spéculation hégélienne le même type d'étrangetés pour le lectorat contemporain ; lire l'excellente introduction : Fabrice Colonna, Ruyer, Paris, Belles Lettres, 2007.

19 Karl Marx, « Postface à la deuxième édition allemande », Le Capital, op. cit., p. 17.

20 Ibid., p. 17-18.

21 Ibid., p. 17.
} 
mente de Marx à l'endroit de la spéculation hégélienne explique sans doute pourquoi des générations de matérialistes ont dévalorisé la valeur réflexive de l'institutionnalisme hégélien : à quoi bon perdre du temps et de l'énergie cérébrale avec une «mystification»? Évidemment, le réalisme ontologique de Hegel reconnaît l'existence d'une dimension suprasensible à même l'être, mais celle-ci, qui a pour essence d'apparaître - et non de rester cachée comme dans l'acception usuelle du mysticisme -, n'est pas concevable sans son articulation avec le moment préalable de l'intuition sensible de la même manière que la valeur ne se saisit pas sans la marchandise ${ }^{22}$. Mais la différence majeure avec Marx, c'est que pour Hegel la dialectique n'est pas anthropocentrique: la production de la totalité du réel n'est pas l'apanage du travailleur, mais de l'être lui-même auquel appartient même le « pire des architectes ». Cela n'a rien de mystérieux : le phénomène de la vie n'est pas une construction sociale.

Ainsi, toute considération honnête envers Hegel devrait partir du surgissement de la vie biologique sur Terre comme fait réel incontournable, car c'est à travers ce phénomène - «mystérieux » si l'on part de la matière inerte - qu'on peut admettre aisément, sans mysticisme, la manifestation empirique de ce que Hegel nomme « idée », « véritable infini » en tant que rapport à soi (l'organisme vivant) à travers son autre (son milieu). C'est quand l'État constitue pour l'esprit le rapport à soi à travers son autre que Hegel le considère « divin 23 » en le comparant - sans réductionnisme biologique - avec un « organisme 24 » vivant. Il n'est pas question ici de légitimation religieuse du monarque ni de théologie politique réactionnaire, bien que, par ailleurs, les religions s'intéressent beaucoup, elles aussi, au phénomène de la vie, d'autant plus que les biologistes, en n'interrogeant plus la vie que dans les laboratoires, ont abandonné la réflexion philosophique pour se concentrer à l'analyse des acides aminés - laissant ainsi le champ libre aux prédicateurs et aux gourous. Le motif, qui explique que la philosophie hégélienne ait pu passer pour « mystique », est qu'elle reconnaît la présence d'un sens, le concept, à même le réel qui se déploie dans la nature, notamment à travers l'orbite des planètes, la géodynamique et la vie biologique, puis dans l'histoire humaine, notamment à travers la réflexivité sociopolitique, les formes symboliques de la piété, de la créativité artistique et de la philosophie. Ainsi, le concept auquel réfère le philosophe spéculatif précède ce dernier dans la mesure où le concept médiatise le développement du cosmos, des embryons et des sociétés, ce qui inclut donc aussi le philosophe qui pense conceptuellement. Le concept exposé dans la philosophie hégélienne est ce qui médiatise toutes les conditions matérielles préalables à la moindre parole, à la moindre pensée. Les matérialistes qui refusent cet aspect de Hegel

\footnotetext{
22 Prescription épistémologique que l'on retrouve dans la Naturphilosophie : « La philosophie ne doit pas seulement être conforme à l'expérience de la nature, mais la naissance et la formation de la science philosophique ont pour présupposition et condition la physique empirique » (G. W. F. Hegel, Werke, t. 9 : Enzyklopädie der philosophischen Wissenschaften in Grundrisse [1830] II : Die Naturphilosophie, Francfort-sur-le-Main, Suhrkamp, 1993, § 246, rem., p. 15).

23 G. W. F. Hegel, Principes de la philosophie du droit, op. cit., § 273 rem., p. 466.

24 Ibid., § 259 rem., p. 430.
} 
ne font qu'amalgamer implicitement le concept à la matière, dont les niveaux d'émergence se trouvent de facto affublés d'attributs divins.

La philosophie hégélienne, qui peut être appelée réalisme de l'idée ou idéalisme objectif, consiste à surmonter rationnellement l'opposition entre foi et savoir en supprimant le réductionnisme inhérent aux croyances religieuses et aux connaissances scientifiques. Cependant, pour Hegel, la philosophie spéculative ne doit jamais contredire les faits scientifiques, bien qu'elle dise plus que ce qui résulte de la science. Si nous mentionnons la centralité du phénomène du vivant chez Hegel, c'est pour démystifier sa philosophie, même si elle se prononce sur les conditions cosmologiques de l'apparition de la vie et sur sa transformation ultérieure en vie humaine de l' « esprit », ou du symbolique, pour prendre un terme plus près de nous ${ }^{25}$. Mais pour l'instant, c'est la césure entre les deux penseurs qui nous intéresse et sur laquelle revient le Marx de la maturité :

Dans son fondement, ma méthode dialectique n'est pas seulement différente de celle de Hegel, elle est son contraire direct. Pour Hegel, le procès de la pensée, dont il va jusqu'à faire sous le nom d'Idée un sujet autonome, est le démiurge du réel, qui n'en constitue que la manifestation extérieure. Chez moi, à l'inverse, l'idéel n'est rien d'autre que le matériel transposé et traduit dans la tête de l'homme ${ }^{26}$.

La première difficulté que l'on rencontre dans cet extrait consiste dans le rejet unilatéral de tenir comme possible l'existence et la connaissance d'une dialectique extrahumaine à même la réalité. Si Marx conçoit l'existence de logiques qui dépassent la seule empirie - ne critique-t-il pas justement l'économie politique qui ne se maintient qu'à la surface des choses ? -, néanmoins il s'agit toujours de constructions humaines. Bien que l'idée soit, selon Hegel, plus qu'un seul « procès de la pensée », on ne peut la qualifier de " sujet autonome », puisqu'il y a existence d'une interdépendance entre les dimensions conceptuelle et réelle. Chez Hegel, le concept s'incarne dans des lieux du réel sous forme d'idée; bien que renfermant en elle-même l'assomption forte d'un fondement subjectif de l'être, l'idée renferme aussi une matérialité en tant que processus, ce que semble concéder le Marx mature avec l'analogie du « démiurge ». Cet aspect matériel est donc incompatible avec l'idée platonicienne, dont la pureté rend

25 La sociologie dialectique de Freitag, dont la théorie du symbolique se fonde sur le rapport d'objectivation concomitant à l'apparition de la vie, est assurément un lieu de rencontre entre Hegel et Marx ; voir Michel Freitag, Dialectique et société, vol. 2 : Introduction à une théorie générale du symbolique, $2^{\mathrm{e}}$ éd., Montréal, Liber, 2011. D'ailleurs, dans des passages cruciaux de son exposition réaliste des modes d'être, Freitag réfère notamment à Ruyer, mentionné plus haut, lequel, malgré sa lecture lacunaire de Hegel et de Marx, a produit une théorie néofinaliste de l'« embryogenèse du monde» à la fois spéculative et informée par les sciences naturelles ; voir Raymond Ruyer, Néo-finalisme, $2^{\mathrm{e}}$ éd., Paris, Presses Universitaires de France, 2012 ; L'Embryogenèse du monde et le Dieu silencieux, Paris, Klincksieck, 2013.

${ }^{26}$ Karl Marx, « Postface à la deuxième édition allemande », Le Capital, op. cit., p. 17. 
inconcevable tout contact avec l'impur matériel qui ne peut qu'être conçu négativement en tant que corruption ou émanation du pur. Au contraire, l'idée chez Hegel consiste dans un processus ternaire renfermant une dimension matérielle, ce qui devient difficile à comprendre pour un lecteur habitué à la définition platonicienne du terme; en tant que résultat d'une manifestation du concept, l'idée n'est donc pas « autonome » : pour s'objectiver dans l'État ou pour jouir d'elle-même dans l'œuvre d'art, il faut absolument qu'agisse l'« esprit subjectif » d'un individu en chair et en os, c'est-à-dire dès lors qu'une politicienne ou qu'un peintre se soit résolu à incarner une cause plus grande que soi. L'interdépendance entre l'idée et l'être humain implique donc une performativité, ce qui place Hegel loin du platonisme et qui devrait amenuiser la portée des railleries feuerbachiennes du jeune Marx à propos de l'inversion du sujet et du prédicat (voir plus bas).

Ainsi, le réalisme ontologique de l'idée, ou l'idéalisme objectif, assume l'existence d'une conceptualité extramentale, indépendante de la subjectivité humaine : l'embryon humain, n'étant pas le producteur libre de son corps, est donc plus proche de la «meilleure des abeilles» que de l' " ex-embryon ${ }^{27}$ » qui est devenu le «pire des architectes ». La philosophie de Hegel transgresse sciemment les bornes de l'idéalisme subjectif moderne en reprenant certains éléments de la philosophie antique et de la théologie chrétienne rejetés par les philosophies du sujet et leurs successeurs nihilistes, qui s'érigent contre la religion tout en refoulant le fait que leur anthropocentrisme dogmatique dérive de la doctrine vétéro-testamentaire de l'imago Dei ${ }^{28}$. Sous cet angle, le matérialisme ouvertement anthropocentrique de Marx reste donc plus proche des philosophies modernes du sujet que ne l'est la Sittlichkeit hégélienne. Ainsi, toutes les utopies anarcho-communistes, d'hier à aujourd'hui, doivent être vues comme les fruits de l'émancipation de la subjectivité rendue effective par les médiations politicojuridiques de l'État et la sécularisation de doctrines judéo-chrétiennes. Contrairement à l'idéalisme objectif qui permet d'entrevoir un humanisme non anthropocentrique - l'être humain étant la cime du développement cosmique de l'idée -, la limitation anthropocentriste de Marx se révèle dans son rapport constructiviste à la nature 29 quand, par exemple, il parle de cerisiers importés «par le commerce ${ }^{30} »$ : les arbres

\footnotetext{
27 Selon Ruyer, la conscience de soi humaine est secondaire à la conscience de soi primaire partagée par tous les organismes vivants, ce qui lui fait dire que la philosophie devrait s'intéresser plus à l'embryologie qu'à la linguistique, qui «n'est qu'un ruisselet secondaire et dérivé de la grande embryogenèse organique ", survenant avec l'apparition de la vie sur Terre (Raymond Ruyer, L'Embryogenèse du monde et le Dieu silencieux, op. cit., p. 231).

28 Alain Supiot, Homo juridicus. Essai sur la fonction anthropologique du Droit, op. cit., p. 35 sq.

29 Voir Frédéric Neyrat, La Part inconstructible de la Terre. Critique du géo-constructivisme, Paris, Seuil, 2016, p. $76 s q$.

$3^{0}$ Karl Marx et Friedrich Engels, L'idéologie allemande, trad. H. Auger et al., Paris, Éditions sociales, 2014, p. 24.
} 
fruitiers ne sont vus que comme réservoirs de valeurs d'usage31, alors que dans la Naturphilosophie de Hegel, qui se veut «libération de la nature $3^{2}$ », ces cerisiers consisteraient avant tout en des organismes médiatisés par le même concept transspatial étant à l'œuvre à la fois, mais différemment, dans le mystère non mystificateur de la photosynthèse et dans la vie éthique.

L'appellation « savoir absolu » pour évoquer la philosophie spéculative renferme à notre époque nominaliste une connotation assez négative, voire ridicule, mais il nous semble possible d'établir un angle d'approche qui permette de surmonter cette situation en reconnaissant, en toute modestie, la présence d'une conceptualité immanente au réel. Il s'agit d'avoir à l'esprit qu'ontologiquement une telle conceptualité puisse être admise comme une évidence non discutable, bien qu'épistémologiquement elle puisse être plus ou moins bien comprise par l'être humain fini qui s'efforce de la concevoir - être philosophe, c'est être ami de la sagesse, et il y a plusieurs degrés possibles d'amitié. Ainsi, cette prudence épistémologique, qui semble parfois avoir fait défaut à Hegel, nous incite à aborder la conceptualité immanente en refusant d'en fixer la présence dans des détails et en misant plutôt sur ses manifestations générales en acceptant une large part de contingences les plus diverses33. Il s'agit donc d'adopter une herméneutique faillibiliste 34 insistant sur l'effort interprétatif, qui peut se tromper, mais qui ne réduit pas la conceptualité immanente du réel à une construction sociale ou à un discours humain, c'est-à-dire à du « matériel transposé et traduit dans la tête de l'homme ». Hegel dépasse la critique de la philosophie moderne du sujet en démantelant le monopole anthropocentriste de la conceptualité, contrairement à l'entendement postmoderne qui s'imagine la critiquer, mais qui, somme toute, s'exécute à l'intérieur d'un anthropocentrisme oscillant entre un perspectivisme, qui inhibe la disposition d'esprit politique, et un constructivisme, qui stimule le développement des forces productives. Ainsi, il n'y a pas de panlogisme chez Hegel qui rendrait possible le parallélisme entre les sphères ontologiques distinctes, contrairement à ce qu'affirme le jeune Marx :

Le même passage est accompli dans la Philosophie de la nature pour passer de la nature inorganique à la vie. Ce sont toujours les

${ }^{31}$ Récemment, l'écosocialiste Joel Kovel a proposé de dépasser l'anthropocentrisme marxiste en reconnaissant une «valeur intrinsèque » à la nature ; voir The Enemy of nature. The end of capitalism or the end of the world?, Londres, Zed Books, 2002.

$3^{2}$ G. W. F. Hegel, Werke, t. 9, op. cit., § 246 add., p. 23.

33 Hegel est conscient du danger que les philosophes encourent lorsqu'ils se prononcent sur des détails non philosophiques, que ce soit quand Platon discute de soins aux nourrissons ou quand Fichte suggère de peindre le portrait des suspects dans leur passeport (Principes de la philosophie du droit, op. cit., p. 131).

34 Pour approfondir la question du paralogisme épistémologiste, qui réduit l'être à la connaissance qu'on en a, on peut lire Roy Bhaskar, "Philosophie et réalisme critique ", dans Le réalisme critique. Une nouvelle ontologie pour la sociologie, dir. Margaret Archer et Frédéric Vandenberghe, Lormont, Bord de l'eau, 2019, p. 47-85. 
mêmes catégories qui prêtent leur âme tantôt pour telle sphère, tantôt pour telle autre. Il n'y a qu'à trouver, pour chaque détermination concrète, singulière, la détermination abstraite qui lui correspond 35 .

Il est vrai qu'en comprenant l'être autour de la notion de vie, de rapport à soi dans l'autre, il puisse y avoir une certaine récurrence conceptuelle, mais ce n'est pas un parallélisme panlogique où tout serait fixé d'avance. Car, si au début de la réception de la philosophie hégélienne, la critique d'un prétendu panlogisme était à la mode ${ }^{6}$, aujourd'hui on est en mesure de comprendre à quel point Hegel concevait qu'il est rationnel qu'il y ait de l'irrationnel. Étant donné que Hegel admettait une part irréductible de contingences, la critique jeune-marxienne du placage de la Logique sur l'État apparaît tout à fait exagérée :

Les différents pouvoirs ne sont donc pas déterminés par leur « nature propre », mais par une nature qui leur est étrangère. La nécessité est tout aussi peu créée par leur propre essence qu'elle n'est démontrée de manière critique. Leur destin est bien plutôt fixé à l'avance par la « nature du concept », il se trouve scellé dans les registres sacrés de la Santa Casa (la Logique). L'âme des objets, ici celle de l'État, est prête, son destin est fixé à l'avance avant son corps, qui n'est à proprement parler qu'une apparence37.

Il faut donc reléguer aux oubliettes la vieille conception d'un " panlogisme » hégélien et la remplacer par une lecture qui insiste sur l'hybridité ontologique permettant de concevoir la composition du réel, d'une part, comme contingente (zufällige) et, d'autre part, comme effective (wirkliche). L'effectivité constitue le réel médiatisé par le concept, tandis que le contingent, le réel non médiatisé par le concept. Tout le réel n'est pas effectif, donc rationnel, même si « ce qui est rationnel est effectif; et ce qui est effectif est rationnel $38 »$ : cette précision ouvre la porte à une critique et une mise à jour du système hégélien pouvant s'effectuer sans devoir jeter le bébé avec l'eau du bain, notamment en nous autorisant, deux siècles plus tard, à soutenir que Hegel a pu avoir forcé la présence d'effectivité là où il n'y avait que contingence; par exemple, dans les questions de la primogéniture aristocratique ou du rôle de la femme dans la famille. La distinction entre réel contingent et effectivité permet ainsi de donner crédit à des critiques précises produites par Marx sans le suivre jusqu'au bout avec son rejet du réalisme hégélien qu'on ne peut plus réduire ni à un panlogisme ni à une révélation divine.

35 Karl Marx, Contribution à la critique de la philosophie du droit de Hegel, op. cit., p. 85.

$3^{6}$ Voir Jean-François Kervégan, « Postface. La politique après l’État et la philosophie », dans Karl Marx, Contribution à la critique de la philosophie du droit de Hegel, op. cit., p. 339.

37 Karl Marx, Contribution à la critique de la philosophie du droit de Hegel, op. cit., p. 90.

$3^{8}$ G. W. F. Hegel, Principes de la philosophie du droit, op. cit., p. 129. 
Il devient dès lors possible d'entrevoir le maintien et le développement théorique et pratique des médiations politico-juridiques, sans devoir justifier ce que l'histoire a rendu injustifiable. Le point de vue hégélien conçoit la réalité comme composée de sphères, qui renferment des degrés plus ou moins intenses d'effectivité ainsi que de la pure contingence soumise aux hasards des déterminismes. Cependant, cette gradation d'effectivité permet de lire Hegel en comprenant que des sphères du réel ou des événements peuvent être dépourvus de concept, par exemple, lors de cataclysmes sismiques ou de déclins historiques, quand il y a stagnation, voire régression de l'historicité du concept dans l'esprit objectif. En dépit de la philosophie de l'histoire chez Hegel comme lieu de manifestation du sens, il n'est donc pas antihégélien de constater que l'historialité humaine renoue parfois avec des logiques sociétales depuis longtemps dépassées ; par exemple, en ce qui concerne le stade historique du règne romain - où Hegel souligne qu'une "différenciation s'accomplit jusqu'au déchirement infini de la vie éthique entre les extrêmes de la conscience de soi privée personnelle et de l'universalité abstraite 39 »-, on constate que cela en dit plus sur la corruption capitaliste de la citoyenneté postmoderne que sur la Sittlichkeit moderne.

Pour interpréter la démarche spéculative de Hegel, qui consiste à saisir les traces du concept se manifestant dans le réel, nous proposons d'admettre à la fois une ontologie réaliste forte et une épistémologie herméneutique faible. Aussi est-il essentiel de déplatoniser l'idée conçue par Hegel en se rappelant qu'il utilise fréquemment l'exemple du germe devenant arbre : à l'instar des êtres vivants, le système solaire et l'État sont des idées, en tant que processus matériels médiatisés par le concept. Quand Hegel considère l'idée-processus comme " véritable infini », il s'agit du rapport à soi d'un sujet à travers son rapport à l'autre. Cela n'a rien de céleste ; d'ailleurs Hegel critique fréquemment les représentations abstraites - religieuses, métaphysiques ou scientifiques - qui réfèrent à un « mauvais infini » : les religions dualistes et le calcul infinitésimal ont en commun la négation abstraite, indéfinie, du fini. Le véritable infini n'est pas le Dieu monarchiste trônant dans les cieux, ni quelque pure unité impassible, mais la vie en tant que processus où le concept dépasse la contingence à travers des étants en mouvement ou des sujets agissants.

Conjointement à cette nécessaire déplatonisation de Hegel, il faut également étendre le même genre de procédé à la conception hégélienne de la religion, bien que ce soit une question compliquée dont la réponse ne demeurera ici qu'au stade de linéament. La religiosité de Hegel reste pour la pensée critique actuelle, où l'athéisme prédomine, un aspect rebutant qui amplifie la portée du réquisitoire que le jeune Marx rédige contre les Principes de la philosophie du droit. Nous devrons ici nous contenter d'affirmer deux choses sur la teneur théologique de l'hégélianisme qui doivent relativiser les critiques habituelles de l'aliénation religieuse. D'une part, Hegel ne réfère jamais à l'immortalité de l'âme ni au Jugement dernier ; les Principes de la philosophie

39 Ibid., § 357, p. 555. 
$d u$ droit se concluent sur une philosophie de l'histoire, en tant qu'interface des institutions sociopolitiques avec l'esprit absolu où la responsabilité de nos actes ou de notre quiétisme sera jugée par un seul tribunal, celui de l'histoire. Donc, la reconnaissance, par Hegel, d'un sens «divin » dans la nature et dans l'histoire humaine - l'État comme Dieu mortel40 - n'implique aucunement le déni de la finitude humaine, donc de la mort comme conclusion définitive de la vie singulière; en cela Hegel semble tout à fait areligieux, plus proche de l'athéisme que de la religion, contrairement à ce que peuvent penser Feuerbach, Marx et la multitude de leurs descendants 41 . Mais puisque la philosophie hégélienne n'est pas anthropocentriste, elle ne fait pas dépendre l'existence de Dieu de l'immortalité de l'âme individuelle. Lorsque Hegel use de l'adjectif «divin», comme synonyme d' "idéel», c'est pour évoquer une sphère de la réalité où s'incarne le concept : le système solaire, la Terre, la santé du corps et la Sittlichkeit sont des manifestations « divines » même si la contingence peut mettre à l'épreuve le concept : extinction du Soleil, collisions d'astéroïde, cancer, tyrannie. Autrement dit, on peut bien admettre que Hegel est religieux, mais lorsqu'il dit que la lecture du journal « est une sorte de prière réaliste du matin » pour prendre connaissance de l'état réel de l'absolu, on n'a pas affaire à une grenouille de bénitier. La religiosité de Hegel ne peut pas être confondue avec le sacrifice de l'intellect qu'évoque Weber lorsque l'entendement scientifique succombe à la croyance religieuse $4^{2}$ : chez Hegel, l'assomption du « divin » consiste plutôt dans l'acte inaugural du penser spéculatif.

Somme toute, il y a un hiatus irréconciliable entre Hegel et Marx : alors que celuici reprend l'idée feuerbachienne que la religion est créée par l'homme, Hegel prétend au contraire que la « religion est une création de l'esprit divin ; [qu']elle n'est pas une invention de l'homme, mais une production de l'agir divin en lui43 ». Mais comme nous l'avons mentionné plus haut, trancher cette question revient, selon la prescription kantienne, à transgresser la limite de l'intuition sensible, indépendamment du choix auquel on se résout. Quant à l'épineuse question de la religion en tant que garante du tiers symbolique nécessaire à la vitalité des institutions, nous pouvons seulement dire que Hegel et Marx sont diamétralement opposés, tandis que des sociologues comme

40 À côté de la parenté avec Hobbes, c'est à la formule oxymorique choisie par Hegel qu'il faut s'arrêter plutôt que sur « Dieu » : d'un point de vue religieux, « Dieu mortel» ne peut être qu'une aberration.

${ }^{41}$ Voici un récit du jeune Heinrich Heine, en visite chez le professeur Hegel : « je regardais avec extase le ciel étoilé, et j'appelais les astres le séjour des bienheureux. Mais le maître grommela en lui-même : "Les étoiles, hum ! hum ! les étoiles ne sont qu'une lèpre luisante sur la face du ciel. - Au nom de Dieu ! m'écriai-je, il n'y a donc pas là-haut un local de béatitude pour récompenser la vertu après la mort ?" Mais Hegel, me regardant fixement de ses yeux blêmes, me dit d'un ton sec : "Vous réclamez donc à la fin encore un bon pourboire pour avoir soigné madame votre mère pendant sa maladie ou pour n'avoir pas empoisonné monsieur votre frère ?" "(Bernard Mabille, Hegel. L'épreuve de la contingence, Paris, Aubier, 1999, p. 270).

42 Max Weber, Le savant et le politique, trad. C. Colliot-Thélène, Paris, La Découverte, 2003, p. 107.

43 G. W. F. Hegel, Vorlesungen über die Philosophie der Religion, t. 1 : Einleitung. Der Begriff der Religion, Hambourg, Meiner, 1993, p. 46. 
Durkheim, Weber et Freitag occupent la position mitoyenne qu'entraîne leur constat paradoxal où les non-croyants qu'ils sont et qui vivent dans les sociétés les plus sécularisées de l'histoire humaine affirment, chacun à sa manière, que la socialité dépend de la croyance collective en une dimension sacrée.

\section{L'inversion du sujet et du prédicat}

La critique la plus incisive que développe Marx dans sa Contribution à la critique de la philosophie du droit de Hegel vise l' « inversion du sujet et du prédicat »; la prise de connaissance de cette critique constitue la pierre de touche qui met l'hégélianisme à l'épreuve, car c'est à cette occasion que le texte de Marx oblige à prendre position entre Hegel et lui. Cette critique de la philosophie spéculative, amorcée par Feuerbach, porte directement sur le thème de la "subsomption" du singulier et du particulier sous l'universel 44 ». S'il en est ainsi, c'est parce que Hegel considère que la présence du concept à même le réel autorise à se poser du point de vue de l'idée ; c'est cette élévation du philosophe qui permet de concevoir dialectiquement la liberté humaine d'après son concept. Pour la dialectique spéculative hégélienne, les individus libres constituent les « accidents », les « représentations », la " figure apparaissante » et l'« effectivité » des " puissances éthiques 45 » substantielles, c'est-à-dire des médiations politico-institutionnelles configurées dans toute société en tant que totalité. C'est du point de vue de l'idée que Hegel écrit au paragraphe 262 des Principes de la philosophie $d u$ droit, paragraphe tant abhorré par Marx, à propos du rapport de la famille et de la société civile à l'État :

L'idée effective, l'esprit qui se scinde lui-même en les deux sphères idéelles de son concept, la famille et la société civile, en tant [qu'elles sont] sa finité, pour devenir, à partir de leur idéalité, esprit effectif, infini pour soi, répartit de ce fait entre ces sphères le matériau de cette effectivité finie qui est la sienne, [à savoir] les individus en tant que multitude, de sorte que cette répartition apparaît, à même l'individu-singulier, médiatisée par les circonstances, l'arbitre et le choix propre de sa destination ${ }^{46}$.

Marx y voit « tout le mystère de la Philosophie du droit et de la philosophie hégélienne en général $47 »$, car ce paragraphe occulterait ce qu'il considère comme le fait de la dépendance de l'État abstrait envers les sphères, présupposées concrètes et antérieures, de la famille et de la société civile :

la spéculation exprime ce fait comme un acte de l'idée, non pas comme l'idée de la multitude, mais comme l'acte d'une idée

44 Karl Marx, Contribution à la critique de la philosophie du droit de Hegel, op. cit., p. 138.

45 G. W. F. Hegel, Principes de la philosophie du droit, op. cit., § 145, p. 316.

46 Ibid., § 262, p. 435.

47 Karl Marx, Contribution à la critique de la philosophie du droit de Hegel, op. cit., p. 83. 
subjective, différente du fait lui-même [...]. L'état de fait d'où l'on part n'est pas saisi en tant que tel, mais en tant que résultat mystique. L'effectif devient manifestation phénoménale; pourtant l'idée n'a pas d'autre contenu que cette manifestation phénoménale 48 .

C'est là le cœur du problème : à l'instar de Hegel, Marx enfreint aussi la prescription épistémologique kantienne étant donné qu'il est autant interdit de prétendre que "l'idée n'a pas d'autre contenu que cette manifestation phénoménale », que de prétendre spéculativement que cette manifestation phénoménale reflète, affirmerait Raymond Ruyer, un " thème mnénique 49 » trans-spatial qui déborde le cadre spatiotemporel de tout organisme en orientant toutefois le développement de celui-ci. Qu'il faille trancher entre existence ou inexistence du concept peut, de prime abord, sembler byzantin, mais si l'on assume que la raison éthicopolitique autorise à surmonter cet écueil de la raison théorique afin de pouvoir opter pour telle dogmatique plutôt que pour telle autre, on comprend que le point de vue de Hegel est entièrement justifié par l'idée de liberté et que c'est pour cette raison qu'on peut rejeter la critique de Marx visant la "subsomption" du singulier et du particulier sous l'universel». Cette critique occulte l'effet libérateur de l'universel sur le singulier, de même que la « subsomption » d'un francophone sous la grammaire et le glossaire de la langue de Molière libère l'expression de ses désirs et de sa créativité, tout en faisant vivre le français. Pour parler comme un Américain, il s'agit d'un win-win result tel que cela va dialectiquement de soi pour la pensée institutionnaliste de l'État.

L'opposition entre le point de vue de Hegel et celui de Marx est exacerbée par la difficulté, déjà mentionnée, que cause la définition ternaire de l'idée. À la lecture de la Contribution à la critique de la philosophie du droit de Hegel, force est de constater la présence d'une distorsion dualiste de la conception ternaire de l'idée : à l'instar de Feuerbach, Marx la considère oppressante envers l'individu en chair et en os en plus d'en être la créature, tandis que Hegel considère l'individu comme « moment » charnel d'un processus qui le dépasse et qui rend possibles sa vie et sa liberté. Il y a donc chez Feuerbach et chez Marx une dualisation platonisante de l'idée, qui est étrangère à la conception trinitaire hégélienne. Quand Hegel dit que "l'idée se scinde en deux sphères », cela correspond analogiquement au constat du linguiste qui déplore que « le français s'appauvrit à la radio »: dans les deux cas, nous sommes en présence d'un spécialiste qui parle au nom de médiations qui se reproduisent, plus ou moins, à l'insu de la conscience des individus, dont la propre existence ne peut plus se maintenir par les seules capacités biologiques de leur corps. Pour Hegel, l'idée est un processus entre différents moments interdépendants qu'occulte complètement la distorsion dualiste de Marx, ce qui a pour effet

\footnotetext{
48 Ibid., p. 83 (trad. modifiée).

49 Raymond Ruyer, L'Embryogenèse du monde et le Dieu silencieux, op. cit., p. 70 sq.
} 
de monter en épingle l'inversion du sujet et du prédicat, où l'idée devient une sorte de macrosujet abstrait parasitant la concrétude de la vie humaine :

C'est précisément parce que Hegel part des prédicats, de la déter-

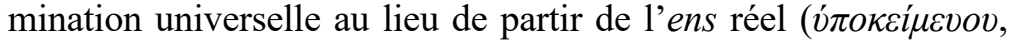
sujet), et qu'il faut pourtant bien qu'un porteur soit là pour porter cette détermination, que l'idée mystique devient ce porteur en question. C'est cela le dualisme, à savoir que Hegel ne considère pas l'universel comme l'essence effective de ce qui est effectivement fini, c'est-à-dire de ce qui a une existence, du déterminé, ou encore qu'il ne considère pas l'ens effectif en tant que sujet véritable de l'infiniso.

Le jeune Marx montre qu'il ne saisit pas le trinitarisme immanent de la philosophie hégélienne lorsqu'il écrit que «[1]e "concept" est le Fils dans 1" "idée", dans Dieu le Père, il est l'agens, le principe déterminant, différenciant. "Idée" et "concept" sont ici des abstractions rendues autonomes ${ }^{11}$ », car chez Hegel le réel sensible, c'est-à-dire empirique, fait partie intégrante de l'idée, qu'on peut se représenter comme rationalisation philosophique d'une innovation théologique chrétienne, qui, en totale opposition au platonisme, se représente Dieu comme totalité vivante, périchorétique. Dans la conception trinitaire, il y a justement interdépendance des trois personnes, ce qui contredit la dualisation opérée par Feuerbach et Marx, car, pour Hegel, le concept, en tant qu'idée logique («Père »), se sensibilise comme être naturel («Fils») et revient à lui-même à travers la structure des médiations sociosymboliques (« Esprit»); cela ne donne pas le même résultat que l'extrait de Marx mentionné ci-haut, d'autant plus que pour Hegel, «sans le monde, Dieu n'est pas Dieu ${ }^{2}$ », donc il n'y a rien dans sa philosophie qui s'apparente au dualisme ontothéologique que l'on retrouve dans la création ex nihilo, comme se le représentent aussi bien l'entendement des créationnistes que celui des théoriciens du Big Bang : l'idée cosmique dont il est question consiste en la liberté vivante qui se déploie concrètement dans la biosphère et dans l'histoire humaine des institutions. Donc, les formulations hégéliennes qui laisseraient entendre une domination du singulier par l'universel s'accordent avec la disposition d'esprit politique nécessaire à la liberté concrète de l'individu en chair et en os : « cette liberté [...] n'est elle-même, tout d'abord, qu'[un] concept, un principe de l'esprit et du cœur, et elle est destinée à se développer en ob-jectivité, en effectivité du droit, de l'éthique et de la religion, ainsi que de la science53 ». L'idée est vie concrète, d'aucune façon représentation abstraite, et son incarnation dépend, lorsqu'elle est dans l'élément de l'esprit, c'est-à-dire dans le symbolique, de la volonté d'un être-là mortel : «L'idée

50 Karl Marx, Contribution à la critique de la philosophie du droit de Hegel, op. cit., p. 104.

51 Ibid., p. 90-91.

$5^{2}$ G. W. F. Hegel, Vorlesungen über die Philosophie der Religion, t. 1, op. cit., p. 213.

53 G. W. F. Hegel, Encyclopédie des sciences philosophiques, t. 3 : Philosophie de l'esprit, trad. B. Bourgeois, Paris, Vrin, 1988, § 482 rem., p. 279. 
apparaît ainsi seulement dans la volonté qui est une volonté finie, mais qui est l'activité de développer celle-là et de poser son contenu se déployant, comme [un] être-là qui, en tant qu'être-là de l'Idée, est [1']effectivité, - [tel est l']esprit objectiff4 ». Ainsi, à l'opposé de ce que prétend Marx lorsqu'il écrit que l' ' idée est transformée en sujet55 » et le sujet réel en prédicat, Hegel conçoit plutôt que le sujet réel est l'idée en tant que processus dont homo sapiens est la particularisation : "Si le savoir de l'Idée [...] est spéculatif, cette Idée elle-même est, en tant que telle, l'effectivité des hommes, non pas l'Idée qu'ils en ont, mais celle qu'ils sont $5^{6} \gg$. Or, selon Marx, une idée ne peut qu'être que ce que les hommes ont en tant que «matériel transposé » dans leur tête, tandis que, selon Hegel, les hommes sont l'idée, en tant que « démiurge du réel 57 ». Dans la philosophie spéculative de Hegel, il n'y a pas de domination de l'abstrait sur le concret, ni de création ex nihilo de l'impur par le pur : toutes ces lectures erronées de l'hégélianisme révèlent une distorsion dualisante comme celle qu'ont induite Feuerbach et Marx, et qui continue à se propager jusqu'à aujourd'hui.

\section{La Constitution comme construction non sociale}

Aussi paradoxal que cela puisse paraître, c'est la conception spéculative du fondement hétéronomique de la liberté humaine qui explique que pour Hegel la Sittlichkeit ne peut pas être une démocratie radicale autofondée, mais plutôt un régime qui, comme la monarchie constitutionnelle 58 , renferme en lui-même des médiations dont la légitimité est sacrée, donc à l'abri des manipulations humaines. Or, cette conséquence politique de la philosophie hégélienne est aberrante pour Marx et la pensée critique contemporaine, qui a développé le réflexe de réclamer toujours plus de démocratie directe à chaque problème rencontré dans la vie sociale. Dès qu'une pensée refuse d'admettre la nécessité ontologique d'un tiers symbolique, seule peut effectivement en résulter l'exigence d'une vraie démocratie : «De même que ce n'est pas la religion qui crée l'homme, mais l'homme qui crée la religion, de même ce n'est pas la constitution qui crée le peuple, mais le peuple qui crée la constitution59 ». Pour Marx, il va de soi que la démocratie se retrouve au sommet de tous les régimes historiques :

La démocratie, c'est l'énigme résolue de toutes les constitutions. Ici, ce n'est pas uniquement en soi, d'après l'essence, mais d'après l'existence, d'après l'effectivité que la constitution se trouve continûment reconduite au fondement effectif qui est le sien, à l'homme effectif, au peuple effectif, et qu'elle est posée comme étant son

54 Ibid., § 482, p. 278.

55 Karl Marx, Contribution à la critique de la philosophie du droit de Hegel, op. cit., p. 81.

$5^{6}$ G. W. F. Hegel, Encyclopédie des sciences philosophiques, t. 3, op. cit., § 482 rem., p. 279.

57 Karl Marx, « Postface à la deuxième édition allemande », Le Capital, op. cit., p. 17.

$5^{8} \mathrm{Hegel} \mathrm{ne} \mathrm{mentionne} \mathrm{que} \mathrm{la} \mathrm{monarchie} \mathrm{constitutionnelle,} \mathrm{un} \mathrm{régime} \mathrm{encore} \mathrm{très} \mathrm{répandu,} \mathrm{notamment} \mathrm{en}$ Europe de l'Ouest où la liberté individuelle et les programmes sociaux sont bien vivaces.

59 Karl Marx, Contribution à la critique de la philosophie du droit de Hegel, op. cit., p. 112 (trad. modifiée). 
œuvre propre. La constitution apparaît pour ce qu'elle est, à savoir un libre produit de l'hommero.

La démocratie moderne, dotée du pouvoir législatif, consiste dans une avancée politique majeure ; elle « est l'essence de toutes les constitutions étatiques [...], elle se rapporte aux autres constitutions à la manière dont le genre se rapporte à ses espèces $^{61} \gg$. La démocratie permet la prise de conscience fondamentale de l'autoproduction de l'organisation sociale par les humains, ce qui justement révèlerait la nature dérivée des régimes traditionnels de la monarchie et de l'aristocratie érigés sur la méconnaissance de cette vérité démocratique.

Ainsi, Marx ne peut que rejeter les justifications ontologiques que l'on retrouve dans les Principes de la philosophie du droit qui voient dans la monarchie constitutionnelle la configuration étatique la plus conforme à l'idée de liberté. En effet, pour Hegel, la concrétisation de la liberté humaine exige de soustraire certaines déterminations constitutionnelles à toute profanation démocratique. Il est nécessaire que l'instituant rencontre des limites, puisque, contrairement à ce qu'en dit Marx, la réalité sociosymbolique n'a pas totalement été créée par les humains, lesquels ne font que reproduire et transformer une construction qui n'est pas complètement la leur62. La posture hégélienne se démarque donc de l'anthropocentrisme constructiviste tout en évitant de sombrer - notamment comme Heidegger et les nazis - dans un romantisme païen de l'originel indifférencié ; il s'agit de conserver les acquis modernes du pouvoir législatif, en acceptant d'assumer que la liberté consiste à transformer ce qu'on n'a pas créé. C'est ainsi que Hegel affirme que « la constitution [d'un État], bien que surgie dans le temps ne [doit] pas [être] regardée comme quelque chose de fabriqué63 ». Chez Hegel, la constitution - qui dépasse le texte constitutionnel en incluant l'esprit d'un peuple, sa culture et sa conscience de soi64 - « est à considérer comme ce qui est divin et persistant et comme située au-dessus de la sphère de ce qui est fabriqué65 ». En effet, selon la perspective hégélienne, les configurations politiques doivent se soumettre à une éthique de la responsabilité éloignée de tout constructivisme anthropocentrique qui rendrait trop plastique la structuration de la société par un État vulnérabilisé dès

\footnotetext{
60 Idem.

61 Ibid., p. 113.

62 Le philosophe Roy Bhaskar admet la même chose en insistant sur la distinction entre créer et transformer; le sociologue Michel Freitag parvient à une conclusion similaire en enracinant le symbolique dans le rapport d'objectivation sensori-moteur, présent dans tout le règne animal, et en concevant les modes de reproduction de la société où une large partie des pratiques humaines reste soumise aux exigences ontologiques du maintien de soi dans l'existence, bien que cela s'effectue en « circuit externe », dirait Ruyer, c'est-à-dire à travers le circuit de la normativité sociosymbolique, qui est plus malléable que la normativité biologique du corps organique.

63 G. W. F. Hegel, Principes de la philosophie du droit, op. cit., § 273 rem., p. 466.

64 Ibid., § 274, p. 466.

65 Ibid., § 273 rem., p. 466.
} 
lors que sa constitution ouvre ses portes aux trafics d'influence, aux conspirations, aux paranoïas collectives ou aux couplages structurels capitalistes.

Pour illustrer comment, à l'encontre de Marx, il est possible de faire preuve de générosité herméneutique envers Hegel, on n'a qu'à considérer la découverte anthropologique du tabou universel de l'inceste, tel que théorisé par Lévi-Strauss. Cet interdit, qui est au fondement de l'humanité, ne peut être compris comme résultat d'une intention humaine, puisque l'humanité présuppose cet interdit et ne peut donc avoir construit cette condition ontologique du processus d'hominisation, à la fois nécessaire et antérieure à toute construction sociale. Qui plus est, la primatologie a récemment renoué avec le fameux effet Westermarck, qui reconnaît dans le contexte présymbolique des primates supérieurs des patterns éthologiques d'évitement de l'inceste ${ }^{66}$. Ainsi, l'effet Westermarck s'avère une norme animale préfigurant la norme symbolique du tabou d'inceste, et il semble donc rationnel d'admettre une part infrahumaine, posée sans l'intervention consciente d'un humain instituant, au sein de la première forme instituée de socialité humaine. Hegel nous a habitués à assumer ce que les sciences sociales tendent à éviter et, si l'on s'accorde pour conférer au tabou universel de l'inceste le statut de mère de toutes les constitutions politiques, il est donc possible de comprendre la part inconstructible des constitutions.

Or, Marx considère contradictoire de priver la capacité législative acquise dans la modernité de la possibilité de s'appliquer à la constitution elle-même :

Le pouvoir législatif n'est pouvoir législatif qu'à l'intérieur de la constitution, et la constitution se trouverait hors de loi si elle se trouvait à l'extérieur du pouvoir législatif. Voilà la collision ${ }^{67}$.

C'est en passant par la nature des choses et des rapports qu[e Hegel] fait ce qu'il ne devrait pas faire d'après la nature de la constitution. Il fait matériellement, factuellement, ce qu'il ne fait pas formellement, légalement, constitutionnellement. Ce faisant Hegel n'a pas levé l'antinomie ; il l'a transformée en une autre antinomie ; il a mis en contradiction la capacité du pouvoir législatif à produire des effets, sa capacité à produire des effets constitutionnels, avec la détermination qu'il reçoit de la constitution. Demeure l'opposition entre la constitution et le pouvoir législatif68.

Comme avec l'inversion du sujet et du prédicat, il y a ici un aspect irréconciliable entre l'anthropocentrisme de Marx, qui insiste sur les conséquences constitutionnelles découlant de la prise de conscience qu'aucune construction humaine ne peut souffrir

66 Voir Bernard Chapais, Liens de sang. Aux origines biologiques de la société humaine, Montréal, Boréal, 2015.

67 Karl Marx, Contribution à la critique de la philosophie du droit de Hegel, op. cit., p. 145 ; les mots soulignés sont en français dans l'original.

68 Ibid., p. 146. 
d'une limitation qui viendrait d'un tiers suprahumain, et l' « idéocentrisme » de Hegel, qui considère qu'une Sittlichkeit est quelque chose de « divin » qui doit se tenir audessus de la mêlée du pouvoir législatif pour garantir la sacralité de l'autonomie individuelle effective.

C'est donc l'affirmation spéculative du sens à même la configuration de l'État qui justifie, selon Hegel, que la monarchie constitutionnelle est le meilleur régime pour garantir l'effectivité de la liberté, tandis qu'elle n'est pour Marx qu'un « hybride malheureux $69 »$. Or, cette hybridité, loin d'être malheureuse d'un point de vue hégélien, est le signe de la nature ternaire de l'organisme étatique et de la robustesse de la liberté qu'il institue. Pour Hegel, la monarchie constitutionnelle permet la réconciliation entre une dogmatique de la constitution et la négativité créatrice $d u$ pouvoir législatif, bien qu'il limite également celui-ci par le contrepoids d'une chambre haute (comme cela d'ailleurs est encore en vigueur dans les pays les plus démocratiques d'Occident). Ainsi, tous les traits antidémocratiques hégéliens sont légitimés par une éthique de la responsabilité sensible au danger qu'un démocratisme sans limites mènerait la liberté humaine à sa perte. C'est parce qu'il ne partage pas l'optimisme anthropologique des libéraux - auxquels il est, sous certains angles, impossible de ne pas inclure Marx - que Hegel considère la démocratie élargie de la multitude comme incompatible avec les institutions de la liberté et qu'il peut donc concevoir en toute vraisemblance la nécessité d'une transmission héréditaire du trône. La majesté du monarque héréditaire - aspect des Principes de la philosophie du droit qui exaspère Marx au plus haut point - est censée protéger les institutions contre l'influence indue d'intérêts particuliers relayés par le parlement. Cela doit également empêcher que la décision politique soit le fruit d'un calcul impersonnel de votes livrant ainsi la destinée de la société à la contingence réifiante du nombre, ce qui risque de détourner chaque citoyen de la Sittlichkeit dès lors qu'il s'aperçoit que sa voix compte comme une goutte d'eau dans l'océan ${ }^{70}$. À travers le charisme stable de la monarchie héréditaire, Hegel considère que la décision politique demeure le fait d'un être humain redevable, en dernière instance, devant le tribunal de l'histoire. Ainsi, la rigidité constitutionnelle de l' « autoritarisme libéral ${ }^{71}$ » de Hegel est à saisir dialectiquement comme inversement proportionnelle à la liberté individuelle que l'État a rendu concrètement possible au sein de la société civile dans les fédérations, les unions, les groupements, les écoles, les communes et autres associations de producteurs libres.

69 Ibid., p. 178.

$7^{70}$ G. W. F. Hegel, Principes de la philosophie du droit, op. cit., § 311 rem., p. 516.

${ }^{71}$ Bernard Bourgeois, « La pédagogie de Hegel » dans G. W. F. Hegel, Textes pédagogiques, Paris, Vrin, 1990, p. 36. 


\section{Les apories de la critique marxienne des médiations "préhistoriques »}

La thèse centrale de Marx est d'affirmer que les Principes de la philosophie du droit favorisent l' «Idée » abstraite, l'universel, au détriment de l'individu concret, le particulier : comme le capital, l'État parasiterait le vivant. Pourtant, on peut constater que plusieurs aspects fondamentaux de l'«éthicité » conçue par Hegel s'approchent fortement des conceptions de l'autonomie individuelle présentes dans la théorie sociologique - de Durkheim à Freitag en passant par Weber. Par-delà leurs différences, ces sociologues partagent le fait d'avoir légué à leur discipline des synthèses qui permettent de reconnaître la pertinence sociologique de divers aspects de la Sittlichkeit, pourtant durement critiquée par Marx. Ainsi, on peut admettre que l' « esprit objectif », l'objet des Principes de la philosophie du droit, consiste, ni plus ni moins, dans la société $7^{72}$ prise comme « structure morale » (Durkheim) ou comme « structure a priori des médiations culturelles-symboliques et politico-institutionnelles » (Freitag), c'està-dire comme structure supra-individuelle qui rend possible la connexion entre faits et valeurs.

La clef herméneutique que nous avons forgée pour comprendre le sens de la Contribution à la critique de la philosophie du droit de Hegel consiste à reconnaître que là où Hegel conçoit une interdépendance entre pratiques subjectives et institutions, Marx conçoit plutôt une dépendance des institutions envers les pratiques. D'un côté, Hegel saisit dialectiquement l'interdépendance entre l'autonomie individuelle et la verticalité politico-institutionnelle, qui est légitimée grâce à une figure symboliquement représentée comme tiers supérieur pourvu de sens - un autrui généralisé dogmatiquement soustrait de la profanation -, et assume ainsi la prise de conscience suivant laquelle une part de la structuration des normes instituées de la Sittlichkeit rend possible la liberté humaine tout en ne relevant pas de quelque construction sociale et exigeant, pour cette raison, une « libre-aliénation » envers ces normes. De l'autre côté, Marx porte un regard plus étroit sur la dialectique entre individu et médiations politicojuridiques en n'insistant que sur la dépendance de ces dernières vis-à-vis de l'individu, dont l'autonomie, plutôt que d'en dépendre, en serait entravée : les médiations, tels l'État, le droit, la religion, etc., sont ainsi négativement appréhendées comme obstacles à éliminer ; elles sont unilatéralement conçues comme abstractions réelles créées par les humains pour dominer ou endurer la domination. Le cœur de la critique marxienne de la philosophie hégélienne réside dans le fait qu'entre médiations politico-juridiques et pratiques individuelles, Marx voit du parasitisme là où Hegel voit du mutualisme.

Par conséquent, nous esquisserons dans les pages qui suivent divers problèmes inhérents à l'anti-institutionnalisme marxien, qui nous permettent aussi de constater que

\footnotetext{
72 Tout comme l'éthicité chez Hegel, le concept sociologique de société englobe État, société civile et famille, et ce, même si parfois on réfère à la société civile, coupée de son adjectif, par exemple, en disant que l'État se pose au-dessus de la société.
} 
la théorie hégélienne de l'esprit objectif s'approche souvent plus de la tradition sociologique que Marx. Dans cette deuxième section, nous aborderons succinctement quatre thèmes : 1) la multitude libre de tout fondement ;2) la société civile et la famille comme présuppositions réelles de l'État abstrait ; 3) le rejet de l'administration publique, et 4) le besoin de dictature et de messianisme comme dispositifs compensatoires.

\section{Pour une multitude libre de tout fondement}

Dans Sur la Question juive, Marx distingue émancipation politique et émancipation humaine. À travers la laïcité de l'État, où cesse la discrimination envers les minorités religieuses, l'être humain « s'émancipe politiquement de la religion, en la faisant sortir du droit public pour la reléguer dans le droit privé73 ». «L'émancipation politique est la réduction de l'homme, d'une part à sa qualité de membre de la société civile, à l'individu égoïste, indépendant, d'autre part au citoyen, à la personne morale 74 ». Pour Marx, cette forme d'émancipation, « qui laisse debout les piliers de l'édifice75 » n'est censée être qu'une étape vers l'émancipation humaine propre à la « révolution radicale $^{76} »$. L'émancipation politique «n'est certes pas la forme ultime de l'émancipation humaine en général, mais [...] la dernière forme de l'émancipation au sein de l'ordre du monde tel qu'il existe jusqu'à présent 77 ». L'émancipation humaine, qui n'exige plus de médiations politiques entravant la socialité, surviendra

lorsque l'homme individuel réel réintégrera en lui le citoyen abstrait et sera devenu comme homme individuel dans la vie empirique, dans son travail individuel, dans ses rapports individuels, un être appartenant à l'espèce, que l'homme aura reconnu et organisé ses forces propres comme forces sociales et ne séparera donc plus de lui la force sociale sous la forme de la force politique ${ }^{78}$.

À l'institutionnalisme de la Sittlichkeit, qui vise l'émancipation politique, Marx oppose donc la révolution radicale devant ériger la " vraie démocratie » porteuse de l'émancipation humaine, une « démocratie contre l'État », directe, non représentative, dans laquelle le pouvoir législatif se réalise en s'abolissant, en se transformant en une « association des producteurs libres ». "La forme politique de l'émancipation» doit revêtir la forme Commune, non la forme république79; comme le précise Étienne Balibar :

c'est la possibilité de penser une pratique politique autonome qui

n'est pas assujettie à une souveraineté, qu'elle soit transcendante

\footnotetext{
73 Karl Marx, Sur la Question juive, trad. J.-F. Poirier, Paris, La Fabrique, 2006, p. 44.

74 Ibid., p. 63.

75 Karl Marx, Contribution à la critique de la philosophie du droit de Hegel. Introduction, op. cit., p. 294.

${ }^{76}$ Idem.

77 Karl Marx, Sur la Question juive, op. cit., p. 44.

78 Ibid., p. 63.

79 Voir Stathis Kouvélakis, «La forme politique de l'émancipation », dans Marx politique, dir. JeanNuma Ducange et Isabelle Garo, Paris, La Dispute, 2015, p. 66 sq.
} 
ou immanente, et qui «institue le social» de façon permanente [...]. Suivant une voie étroite entre l'anarchisme [...] et un socialisme du travail qui veut résorber le politique dans « l'administration des choses » [...], Marx voudrait faire de l'émancipation du sujet populaire le lieu public permanent de son autoconstitution, le surgissement de la dimension «générique » de l'existence humaine ${ }^{80}$.

Selon Marx, à l'aune de la révolution, la suppression des médiations politico-juridiques « ne peut consister qu'en ceci que l'intérêt universel devienne effectivement [...] l'intérêt particulier, ce qui n'est possible que par le fait que l'intérêt particulier devient effectivement l'universel ${ }^{81} \gg$. Le remplacement révolutionnaire de l'État par une organisation sociale indique que la sociabilité est donc postulée par Marx comme un a priori du genre humain qui ne nécessite pas la médiation d'un tiers symbolique garantissant que cette sociabilité se déploie parallèlement à l'autonomie de l'individu empirique. À partir de là, il devient possible d'entrevoir que « dans la vraie démocratie l'État politique disparaîtrait ${ }^{82}$ ». C'est ainsi que Marx oppose le social au politique ${ }^{83}$ : ni État ni anarchie, l'idéal consiste en une organisation de la sphère sociale se soumettant les fonctions de l'État. L'association des producteurs libres à travers l'administration démocratique de la puissance technico-économique.

Autrement dit, nous voilà devant une querelle des abstractions : tandis que, de son côté, Marx affirme que l'«abstraction de l'État en tant que tel ressortit seulement à l'époque moderne parce que l'abstraction de la vie privée ressortit seulement à l'époque moderne 84 », du côté de Hegel, c'est plutôt l'incarnation immédiate - sans médiation instituée - de l'universel dans le particulier qui constitue le summum de la pensée abstraite. Marx peut bien admettre que l'« abstraction de l'État politique est un produit moderne $85 »$, cependant, il ne devrait pas échapper au regard sociologique que la « vraie démocratie », dont il parle, en est de toute évidence également le produit. Elle en est le produit, car seul un esprit moderne peut engendrer une telle représentation révolutionnaire comme celle de Moses Hess reprise par Marx, qui met en scène un «monde préhistorique » de la domination dont le destin est d'être remplacé par un «monde historique » dépourvu de domination ${ }^{86}$.

80 Étienne Balibar, «Le moment messianique de Marx », Revue germanique internationale, $\mathrm{n}^{\circ}$ 8, 2008, p. 154.

${ }^{81}$ Karl Marx, Contribution à la critique de la philosophie du droit de Hegel, op. cit., p. 137.

82 Ibid., p. 114.

83 Voir Franck Fischbach, Philosophies de Marx, op. cit., p. 84-91.

84 Karl Marx, Contribution à la critique de la philosophie du droit de Hegel, op. cit., p. 115.

85 Idem.

86 Moses Hess, « Les derniers philosophes », cité dans Franck Fischbach, Philosophies de Marx, op. cit., p. 102. 
Après Marx, la sociologie naissante révèle d'emblée une forme d'inquiétude face aux transformations modernisatrices provoquées par la conjonction des révolutions politiques et industrielles. On peut admettre que Durkheim et Weber vont ainsi accorder plus de valeur à l'héritage politique et idéologique des sociétés traditionnelles, notamment à cause de cette inquiétude que suscitent en Occident des bouleversements qui se succèdent sans arrêt et qui menacent l'intégration sociétale. "La sociologie, mentionne Freitag, se présente donc sur la scène historique-idéologique comme une théorie de la dimension d' "intégration" de la société, de l'existence réelle de la société comme "totalité sui generis" irréductible aux seules dimensions politiques et économiques : tous les classiques, sauf Marx [...], en témoignent ${ }^{87} \gg$.

$\mathrm{Au}$ contractualisme moderne, qui présuppose un optimisme anthropologique et auquel il faut rattacher l'idéal communiste de l'«association des producteurs libres ", Weber préfère la lucidité sociologique qu'il attribue à la pensée traditionnelle, qui concevait de manière pessimiste le monde dans lequel évoluent les institutions politiques.

Même les premiers chrétiens savaient très précisément que le monde est gouverné par des démons, que quiconque se commet avec la politique, c'est-à-dire avec le pouvoir et la violence comme moyens, conclut un pacte avec des puissances diaboliques, et que, pour ce qui est de son action, il n'est pas vrai que le bien ne puisse engendrer que du bien, et le mal que du mal, mais que c'est souvent le contraire. Quiconque ne voit pas cela est en fait un enfant du point de vue politiques8.

Or, le pari de Marx s'avère complètement indifférent à ce type de phronésis dans la mesure où il conçoit une organisation sociale faisant table rase du monde préhistorique et de ses démons. Marx se représente une sociabilité humaine qui pourra se déployer librement sitôt débarrassée des abstractions étatiques parasitaires. Et ce pari est d'autant plus risqué que la disparition de l'État doive se faire politiquement, donc avec la violence comme moyen.

En ne reconnaissant pas l'interdépendance entre pratique individuelle et médiation politico-juridique, Marx néglige une autre caractéristique, qui se trouve au cœur de l'institutionnalisme, antique et moderne, qui sera ultérieurement reprise de diverses façons en sociologie : l'existence de la démocratie implique l'intégration des sphères différenciées de la pratique que rend possible sur le plan idéologique le partage de

\footnotetext{
87 Michel Freitag, «Les sciences sociales contemporaines et le problème de la normativité », Sociologie et sociétés, vol. 19, $\mathrm{n}^{\circ} 2,1987$, p. 30.

88 Max Weber, Le savant et le politique, op. cit., p. 196-197. Dans le même ordre d'idée, Freud critiquait en 1930 l'optimisme anthropologique en faisait appel aux médiations : « La culture doit tout mettre en œuvre pour poser des barrières aux pulsions d'agression des hommes » (Sigmund Freud, Le Malaise dans la culture, trad. D. Astor, Paris, Flammarion, 2010, p. 134).
} 
représentations collectives qui unifient le peuple, ou la nation, par-delà les différences sociales. Ainsi, la médiation réciproque du peuple et du pouvoir, à défaut de quoi les individus amalgamés ne forment qu'une multitude dont la capacité d'action coordonnée est sociologiquement considérée comme insuffisante : la volonté de tous (de la multitude, de la foule), selon Rousseau, n'équivaut pas à la volonté générale (du peuple, de la nation) ${ }^{89}$. Et, pour Hegel, il n'y a rien de pire qu'une société où les médiations politiques, juridiques et sociales deviennent inopérantes faute de peuple ; en effet, il faut

que les intérêts particuliers des communes, des corporations et des individus ne s'isolent pas ou, plus encore, afin que les individussinguliers n'en viennent pas à offrir l'aspect d'une multitude et d'un amas, [et] n'en viennent pas de ce fait à une opinion et à un vouloir inorganique et à une pure et simple violence de masse à l'encontre de l'État organique9o.

Exonéré des obligations de la médiation instituée, le peuple devient multitude, tout comme le monarque devient tyran, puis sombre dans le despotisme qui « définit, de manière générale, l'état d'absence de loi, celui où la volonté particulière [...] vaut en lieu et place de la loi91 »; la démocratie sans dêmos est une aberration, aussi bien théorique que pratique, pour signifier l'ochlocratie. Alors que Hegel conçoit l'interdépendance entre l'exercice normal du droit et l'identité du peuple, Marx, ne considérant que la dépendance de celui-là envers celui-ci, naturalise l'existence du peuple en le confondant avec la multitude92.

\section{La société civile et la famille comme présuppositions de l'État abstrait}

En plus d'être superflue pour l'existence du peuple, la médiation étatique de la société civile et de la famille est dénigrée par Marx. Après avoir accentué la toute-puissance étatique en attribuant à Hegel que «le rapport effectif de la famille et de la société civile à l'État est saisi sous la forme d'une activité interne, imaginaire 93 », Marx va ensuite reprendre l'idée que l'État abstrait, parasitaire, dépend unilatéralement de la famille et de la société civile, lesquelles représentent le concret, le lieu de la vie, de l'énergie vitale.

\footnotetext{
89 Voir Jean-François Kervégan, «Peuple », dans Dictionnaire de philosophie politique, dir. Philippe Raynaud et Stéphane Rials, Paris, Presses Universitaires de France, 1996, p. 462.

90 G. W. F. Hegel, Principes de la philosophie du droit, op. cit., § 302, p. 504-505.

91 Ibid., § 278 rem., p. 472.

92 Néanmoins, l'idée d'un amas d'individus asociables incompatible avec les exigences de la vie collective existe dans le marxisme où l'on peut percevoir une analogie entre le rapport peuple/multitude et le rapport prolétariat/Lumpenproletariat.

93 Karl Marx, Contribution à la critique de la philosophie du droit de Hegel, op. cit., p. 81 (trad. modifiée).
} 
La famille et la société civile sont les présuppositions de l'État; ce sont elles qui sont à proprement parler actives; mais dans la spéculation c'est tout l'inverse94.

[F]amille et société civile sont des parties effectives de l'État, des existences spirituelles effectives de la volonté; elles sont des modes d'existence de l'État ; famille et société civile se font ellesmêmes État. Elles sont ce qui donne l'impulsion95.

Or, d'un point de vue hégélien, si l'État a effectivement besoin de la société civile et de la famille, celles-ci ont besoin réciproquement de l'État. Par exemple, l'existence matérielle des institutions sociales de la société civile doit être garantie par l'État, juridiquement ou fiscalement, tandis que l'État a besoin notamment du rôle formateur des institutions sociales afin de faire coïncider l'intérêt universel avec l'intérêt particulier - l'école obligatoire, les syndicats, les fédérations sportives, les églises, etc 96 . Ainsi l'assomption hégélienne de la verticalité politique s'effectue à travers un tissu dense de médiations qui partent de la famille et des instances décentralisées de la société civile jusqu'au sommet de l'État; il s'agit donc, comme l'écrit Bernard Bourgeois, d'une « auto-administration de sphères qui doivent, dans leur particularité, rester subordonnées à l'intérêt supérieur de l'État, et cela pour leur propre bien 97 ».

\section{Le rejet de l'administration publique}

Un troisième élément problématique chez Marx consiste en la critique de la bureaucratie. Pour Hegel, l'acceptation collective du besoin de déléguer des tâches administratives à une " corporation » constituée de fonctionnaires sélectionnés sur la base de leurs connaissances, est une exigence rationnelle. Il s'agit justement d'un exemple où se distingue le peuple de la multitude, de la foule ou de la populace : pour cause de déni des médiations politico-juridiques, les atomes dépolitisés de la multitude présupposent «de la part du gouvernement une volonté mauvaise ou moins bonne98", contrairement aux citoyens politisés du peuple disposés à faire confiance à l'« état universel» des fonctionnaires. À l'opposé de Hegel, Marx ridiculise les hauts fonctionnaires comme « les jésuites de l'État et les théologiens de l'État 99 », les rabaissant ainsi au niveau " préhistorique » d'un pouvoir traditionnel. La bureaucratie s'avère

\footnotetext{
94 Idem.

95 Ibid., p. 82 (trad. modifiée).

${ }^{96}$ La centralité du thème hégélien de la médiation réciproque de la société civile et de l'État a été soulignée dans plusieurs écrits toujours lumineux de Jean-François Kervégan : L'effectif et le rationnel. Hegel et l'esprit objectif, Paris, Vrin, 2007 ; Hegel, Carl Schmitt. Le politique entre spéculation et positivité, Paris, Presses Universitaires de France, 1992.

97 Voir Bernard Bourgeois, La pensée politique de Hegel, Paris, Presses Universitaires de France, 1969, p. 131.

98 G. W. F. Hegel, Principes de la philosophie du droit, op. cit., § 301, rem., p. 503.

99 Karl Marx, Contribution à la critique de la philosophie du droit de Hegel, op. cit., p. 136.
} 
incompatible avec la « vraie démocratie », puisque celle-ci, écrit Emmanuel Renault, se définit « comme le processus niant continûment ce qui dans l'État ne se réduit pas à l'expression actuelle de la volonté du peuple [...] [d]'où la nécessité d'une suppression (Aufhebung) de la bureaucratie ${ }^{100} »$.

C'est pour cette raison que la Contribution à la critique de la philosophie du droit de Hegel renferme un florilège de diatribes antibureaucratiques. Mais il faut comprendre que le procédé rhétorique qu'utilise Marx pour juger la conception hégélienne de l'administration publique consiste d'abord à poser son propre point de vue communiste (donc non réel, seulement à venir) où l'intérêt universel devient effectivement l'intérêt particulier quand l'intérêt particulier devient effectivement l'universel'101 pour l'ensemble de la multitude. Ensuite, Marx va constater que le réel empirique est rempli de mesquinerie, de passe-droit, de corruption : conséquemment, les Principes de la philosophie du droit ne sont qu'une légitimation mystificatrice du statu quo politique. Alors qu'à l'instar de Hegel la sociologie tend à reconnaître comme inévitable que les sociétés différenciées soient dotées d'une élite administrative détentrice de savoirs spécialisés, Marx dénonce que l'" esprit universel de la bureaucratie [soit] le secret, le mystère, gardé de l'intérieur d'elle-même par la hiérarchie et de l'extérieur par le caractère fermé de la corporation ${ }^{102}$ ». Ainsi, Marx va plutôt insister sur le fait avéré, mais collatéral, que les fonctionnaires, malgré la finalité universelle de leur tâche, s'adonnent à des bassesses pouvant être motivées par le simple désir d'enrichissement personnel ou par la névrose obsessionnelle : « cependant, le spiritualisme devient un matérialisme crasse, le matérialisme de l'obéissance passive, de la foi en l'autorité, du mécanisme d'une activité formelle fixe, de principes, de conceptions, de traditions fixes ${ }^{103} \gg$. On pourrait ici rétorquer à Marx qu'il idéalise à son tour la multitude en imaginant que le particulier puisse incarner spontanément l'universel. Ainsi, la reconnaissance hégélienne de la nécessité d'une fonction publique compétente à laquelle est articulée la dimension contrafactuelle, où des serviteurs de l'État se dédient au bien commun, est tournée au ridicule dans de nombreuses pages du manuscrit de Kreuznach.

Or, ce qu'il faut retenir de la critique marxienne de la bureaucratie, c'est qu'elle part du projet de sa suppression future, où la multitude pensera et agira en faveur du bien de tous sans contraintes étatiques, tandis que la sociologie qui compare entre elles des sociétés historiques du « monde social animal » renonce à l'idéal de la désaliénation en se contentant de l'évolution graduelle vers moins d'aliénation. Par exemple, lorsque Weber construit l'idéaltype de la bureaucratie moderne, il la compare à ce qui a déjà existé jadis ; il se contente, d'une certaine manière, de l'avancée que constitue

100 Emmanuel Renault, «Le Droit dans la Critique de la philosophie hégélienne du droit », dans Marx démocrate. Le Manuscrit de 1843, dir. Étienne Balibar et Gérard Raulet, op. cit., p. 30.

${ }^{101}$ Karl Marx, Contribution à la critique de la philosophie du droit de Hegel, op. cit., p. 137.

102 Ibid., p. 136.

103 Ibid., p. 137. 
le fait de " la "séparation" des fonctionnaires et des moyens de fonctionnement104 », car c'en était différent dans les cités de la Renaissance où l'administration publique échouait à quelques membres de familles riches, indépendants de fortune, qui n'avaient donc pas besoin de salaires et qui manifestaient un intérêt sincère à s'occuper de l'administration des choses, notamment de celles qui avaient une incidence sur leur patrimoine. Également, Pierre Bourdieu, dans deux articles éclairants, montre que la centralisation de l'État a lieu en opposition aux réseaux féodaux dans l'antagonisme qu'alimente le roi contre les membres de sa propre famille et de la noblesse, dès lors qu'il décide de constituer son état-major sur la base des compétences juridiques, financières et administratives des roturiers et des juifs, étrangers à sa parenté et à qui il préfère accorder sa confiance au nom de la raison d'État ${ }^{105}$.

\section{Le besoin de dispositifs compensatoires : dictature et messianisme}

Chez Marx, seule compte la première négation dialectique, moment que la perspective hégélienne considère comme anté-spéculatif, car le penser spéculatif exige une deuxième négation en tant qu'affirmation du sens. La deuxième négation permet notamment à Hegel de réconcilier histoire des institutions politiques et sens cosmique, ce que Marx considère comme une mystification. Selon ce dernier, la conséquence politique de la dialectique spéculative, «mystifiée », est la légitimation du statu quo préhistorique de l'institué, tandis que la forme dialectique qu'il propose s'avère, au contraire,

un scandale et une abomination pour les bourgeois et leurs porteparole doctrinaires, parce que dans l'intelligence positive de l'état de choses existant elle inclut du même coup l'intelligence de sa négation, de sa destruction nécessaire, parce qu'elle saisit toute forme faite dans le flux de mouvement et donc aussi sous son aspect périssable, parce que rien ne peut lui en imposer, parce qu'elle est, dans son essence, critique et révolutionnaire ${ }^{106}$.

Ainsi, Marx affiche un optimisme typiquement moderne éloigné de toute phronésis, sans doute traitée comme une vieillerie de réactionnaires angoissés. "Négation", « destruction », « flux », « critique » forment le topique d'une dialectique servant de rasoir à une révolution indifférente aux risques sociologiques que génère une négativité autofondée, libre de toute hétéronomie d'un tiers symbolique. Si ces risques sont bien réels, ce n'est pas parce que la dialectique marxienne scandalise les bourgeois, c'est surtout parce qu'elle sert à légitimer la destruction capitaliste de la société comme

104 Max Weber, Euvres politiques. 1895-1919, trad. É. Kauffmann et al., Paris, Albin Michel, 2004, p. 466.

105 Pierre Bourdieu, «Esprits d’État», Actes de la recherche en sciences sociales, vol. 96, n 1, 1993, p. 49-62 ; « De la maison du roi à la raison d'État», Actes de la recherche en sciences sociales, vol. 118, $\mathrm{n}^{\mathrm{o}} 1,1997$, p. 55-68.

106 Karl Marx, « Postface à la deuxième édition allemande », Le Capital, op. cit., p. 18. 
étape nécessaire de la révolution. En effet, dans le Manifeste, Marx et Engels endossent de manière désinvolte un rôle de démiurge en justifiant une ruse de la raison suivant laquelle les capitalistes sont agis par la finalité communiste : « Les armes dont la bourgeoisie s'est servie pour abattre la féodalité se retournent à présent contre la bourgeoisie elle-même. Mais la bourgeoisie n'a pas seulement forgé les armes qui lui donneront la mort; elle a aussi produit les hommes qui manieront ces armes - les travailleurs modernes, les prolétaires ${ }^{107} \gg$.

Croire que le capitalisme va laisser ses propres contradictions avoir le dessus, c'est révéler au grand jour une mécompréhension de la signification ontologique de l'institution politique. D'ailleurs, le mouvement ouvrier a sauvé à plusieurs reprises le capitalisme de lui-même quand il devenait trop incompatible avec la vie des travailleurs, source de la valeur. Et, la mécompréhension marxienne de la signification ontologique de l'institution politique se révélera également dans la praxis qui, pour réaliser la révolution, devra user immodérément de dispositifs compensatoires : l'État dictatorial et l'espérance messianique.

Le projet révolutionnaire visant une sortie politique du politique consiste à faire du politique le moyen de sa propre abolition. Pour un sociologue lucide comme Weber, il est tout à fait aberrant de vouloir l'abolition du monopole de la violence à l'issue d'une guerre civile, c'est-à-dire d'en appeler « à la violence ultime qui instituerait un état où toute violence serait anéantie ${ }^{108} \gg$. En effet, n'importe quel lecteur débutant de Spinoza sait que « tout être persévère dans son être » et qu'il est alors étrange de priver ainsi l'État de conatus. Exiger de la part des administrateurs du monopole de la violence, militaires et policiers, qu'ils rendent les armes à la multitude armée apparaît sociologiquement comme une rêverie : « Le diable est vieux [...]. Vieillis toi-même pour le comprendre ${ }^{109} \gg$. Le projet marxien de suppression politique de la domination instituée est une véritable contradiction aussi bien en théorie qu'en pratique.

En faisant miroiter une société dépourvue de la verticalité inhérente à la structure des médiations politico-juridiques, le marxisme a suscité de fausses espérances qui ont été dramatiquement déçues, ce qui devrait contribuer au discrédit de la Contribution à la critique de la philosophie du droit de Hegel du jeune Marx. Il ne faut aucunement écarter l'hypothèse suivant laquelle les tyrannies sanglantes exercées en Union soviétique ou en Chine constituent le résultat expérimental d'une praxis qui a été mal inspirée par une théorie inconsistante de la domination instituée ; comme l'écrit le philosophe Denis Collin, qui considère à juste titre le politique comme «le point aveugle » du marxisme, "Lénine disait que le gouvernement ouvrier, c'est la cuisinière au gouvernement, mais il se réalisera en mettant la police politique dans la

107 Karl Marx et Friedrich Engels, Le Manifeste communiste, trad. M. Rubel et L. Evrard, Euvres, t. 1, Paris, Gallimard, coll. « La Pléiade », 1963, p. 168.

108 Max Weber, Le savant et le politique, op. cit., p. 195.

109 Ibid., p. 203. 
cuisine des appartements communautaires ${ }^{110} \gg$. Des dizaines de millions de morts pour constater qu'on a déshabillé Pierre pour habiller Paul : voilà une tragique illustration de ce que Hegel nomme une contradiction d'entendement, laquelle survient quand une conscience réalise l'inverse de ce qu'elle vise.

La signification et l'usage de la dictature du prolétariat, affirme de son côté Étienne Balibar, " résument les problèmes que pose le marxisme comme théorie politique ${ }^{111}$ ", d'autant plus qu'elle est mentionnée rarement dans les écrits de Marx et d'Engels « au regard du problème crucial qu' [elle] désigne : celui de la transition révolutionnaire112 ». La réflexion sur ce dispositif compensatoire, qui occupe le pôle diamétralement opposé à celui de l'" association des producteurs libres », s'est amorcée quand le mouvement révolutionnaire fit l'expérience du principe de réalité, dès les épisodes de répression en 1848, et s'approfondira après l'épreuve de la Commune de Paris - pourtant considérée par Marx comme la " forme positive de la révolution ${ }^{113}$ » - qui fut anéantie rapidement dans la violence. Ce sont ces traumatismes historiques du mouvement ouvrier qui ont contraint Marx à consolider la stratégie de la transition révolutionnaire du prolétariat afin qu'il s'organise en classe dominante, et ce, en dépit de la contradiction découlant du fait que les rapports de classes en tant que tels renvoient à la synthèse sociale capitaliste censée être supprimée par la révolution ${ }^{114}$. Avec la dictature du prolétariat, Marx échafaude la théorie « d'un pouvoir politique qui, pour la première fois dans l'histoire, aurait pour condition l'affaiblissement de l'appareil d'État, ou mieux la lutte contre son existence même ${ }^{115}$ ». L'histoire $\mathrm{du} \mathrm{XX}^{\mathrm{e}}$ siècle a réfuté cette solution mystificatrice, qui devait compenser l'anti-étatisme que l'on retrouve dans la Contribution à la critique de la philosophie du droit de Hegel. Tel n'a-t-il pas été le prix à payer pour la praxis anticapitaliste, fermée à la Sittlichkeit, qui est restée crispée à la première négation?

Parallèlement à la question de l'État, celle de la religion semblait définitivement réglée chez Marx, puisqu'il s'agit clairement d'une création humaine qui sert à

110 Denis Collin, Comprendre Marx, Paris, Armand Colin, 2e éd., 2009, p. 217.

111 Étienne Balibar, «Dictature du prolétariat», dans Dictionnaire critique du marxisme, dir. Gérard Bensussan et Georges Labica, Paris, Presses Universitaires de France, 1982, p. 324.

112 Idem.

113 Voir Stathis Kouvélakis, « La forme politique de l'émancipation », op. cit., p. 70-71.

114 Comme l'écrit justement Robert Kurz, « une classe définie a priori par le capitalisme même, qui se réclame précisément de sa position dans le capitalisme telle qu'elle lui incombe déjà à son insu, ne peut être le moteur d'un mouvement social révolutionnaire " (dans Lire Marx. Les principaux textes de Karl Marx pour le XXI siècle, trad. H. et L. Steinberg, Paris, Balustres, 2002, p. 366). Néanmoins, avec son opposition radicale aux médiations politico-juridiques, Kurz - tout comme l'ensemble de la Wertkritikretombe, à l'instar du Marx «ésotérique », dans l'abstraction préhégélienne de l'optimisme anthropologique des Lumières mettant en scène des individus s'associant librement avec leurs alter ego ; voir JeanFrançois Filion, « Prolégomènes à une analyse comparative de la sociologie dialectique de Freitag et de la Wertkritik», La tyrannie de la valeur, dir. Éric Martin et Maxime Ouellet, Montréal, Écosociété, 2014, p. $235-265$

115 Étienne Balibar, « Dictature du prolétariat », op. cit., p. 327. 
dominer ou à endurer la domination : la révolution communiste rendra caduque cette aliénation. Évidemment, l'hégélianisme prête flanc à la critique athée puisqu'il s'assume comme conscience de soi de l'absolu présupposé connaissable. Or, la teneur théologique de la philosophie spéculative ne peut être traitée de la manière habituellement utilisée par les athées pour critiquer la religion. Chez Hegel, l'idée ternaire comprend en elle-même l'humain en tant que personne matérielle, sensible et mortelle, qui participe au processus périchorétique se manifestant dans l'histoire. Ainsi, le sens de l'histoire, selon Hegel, se constate dans l'accroissement de la liberté ; les actions et les institutions humaines sont jugées par le tribunal de l'histoire ; les êtres humains peuvent incarner, ou pas, les critères de la liberté à travers leurs transformations de la société ; l'État a pour finalité de garantir les conditions de possibilité de la jouissance de l'absolu avec lui-même en laissant vivre son autre : la famille et la société civile, où s'éprouvent l'amour, l'amitié, la création artistique, la croyance religieuse et la connaissance philosophique.

Or, en dépit de son athéisme officiel et de ce qu'en disent ses épigones, on sait aujourd'hui qu'il y a implicitement dans la pensée de Marx des traits théologiques, notamment à travers sa philosophie de l'histoire. Toute philosophie de l'histoire qui assume le déploiement temporel d'un sens renferme nolens volens une dimension théologique. Depuis la publication du classique de Karl Löwith en 1949, Histoire et Salut ${ }^{116}$, il ne fait plus de doute que la pensée de Marx soit empreinte de théologie, ce qui donne une autre saveur aux sarcasmes visant le mysticisme hégélien. Il est désormais établi que Marx reprend la prophétie «judéo-cabalistique117》 de Moses Hess suivant laquelle les derniers, qui ont vécu dans le «monde humain animal», seront les premiers dans le futur «monde humain social ». C'est dans cet ordre d'idées que dans un autre texte éclairant, Étienne Balibar affirme que «[c]omme dans la Kabbale, et particulièrement dans les variantes "utopiques révolutionnaires" du messianisme juif, la résolution de l'injustice historique est conçue comme une recréa-

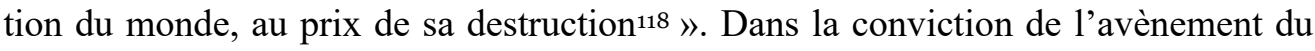
communisme réside donc une conception théologique de l'histoire suivant laquelle les souffrances qu'inflige le capitalisme seront suivies par les bains de sang purificateurs du jugement dernier de la Révolution et laisseront place à la paix perpétuelle d'une vie quotidienne édénique s'accomplissant sans État, sans police, sans travail, sans argent, où le néo-sujet communiste pourra se dire à lui-même que « la société réglemente la production générale, ce qui crée pour moi la possibilité de faire aujourd'hui telle chose, demain telle autre, de chasser le matin, de pêcher l'après-midi, de pratiquer l'élevage le soir, de faire de la critique après le repas, selon mon bon plaisir ${ }^{119}$ ». Ainsi, Marx

116 Karl Löwith, Histoire et Salut. Les présupposés théologiques de la philosophie de l'histoire, Paris, Gallimard, 2002, p. 59.

117 Gérard Bensussan, Moses Hess. La philosophie, le socialisme, Paris, Presses Universitaires de France, 1985, p. 18-19.

118 Étienne Balibar, « Le moment messianique de Marx », op. cit., p. 149.

119 Karl Marx et Friedrich Engels, L'idéologie allemande, op. cit., p. 32 ; nous soulignons. 
réactive « le mythe biblique de l'élection libératrice : l'esclavage "radical" se renverse en mission rédemptrice d'un "peuple du peuple" œuvrant pour toute l'humanité120 ».

\section{Conclusion}

Si le messianisme marxien diffère du mysticisme hégélien, il a cependant accompli fidèlement, à l'époque où il était encore à la mode, la vertu dynamogénique que Durkheim reconnaît à la religion, étant donné que «l'arme de la critique ne peut pas remplacer la critique des armes ${ }^{121} »$. En effet, lorsque la " philosophie trouve dans le prolétariat ses armes matérielles » et «le prolétariat trouve dans la philosophie ses armes intellectuelles ${ }^{122} »$, il faut vraiment qu'intervienne la vertu dynamogénique qui justifie au nom d'un absolu que l'histoire acquittera celui qui a donné la mort pour la révolution ou glorifiera la mémoire de celui qui a donné sa vie pour elle. Mais l'époque où les révolutionnaires étaient des hégéliens inconscients d'eux-mêmes - car « rien de grand ne s'est produit dans le monde sans passion ${ }^{123}$ »- en activant les dispositifs compensatoires que la praxis avait jugés nécessaires d'inventer pour pallier les défaillances théoriques de l'anti-institutionnalisme jeune-marxien - c'est-à-dire en mettant au point les détails de la dictature du prolétariat avec la foi d'agir conformément au sens de l'histoire - est aujourd'hui révolue. Les passages à l'acte qu'a permis cette foi ont connu le destin funeste du néant. Il ne nous semble pas exagéré de prétendre que le désintérêt théorique et pratique du marxisme envers l'institutionnalisme - les réflexions ponctuelles sur la dictature du prolétariat ne pouvant se substituer au concept de Sittlichkeit-n'est pas étranger au fait que les passages à l'acte révolutionnaire, libres de fondement extrahumain, car portés par l'imaginaire de la toute-puissance autofondée, aient révélé leur ineffectivité. De nos jours, le marxisme a abandonné les dispositifs compensatoires de la dictature du prolétariat et de l'eschatologie communiste qui, en dépit de leurs défectuosités, comportaient instinctivement des éléments institutionnalistes nécessaires à toute praxis soucieuse de performativité dans la lutte à finir contre le capital.

Depuis la chute du mur de Berlin, la critique radicale adhère massivement à une idéologie libertaire souvent nourrie par la pensée de la déconstruction visant les rapports de pouvoir ${ }^{124}$. La pensée critique contemporaine prend pour acquise la supériorité de l'instituant aux dépens de l'institué, la conception associative du lien social et la démocratie participative, ce qui s'accorde harmonieusement avec l'antiinstitutionnalisme du jeune Marx. Or, n'est-il pas légitime de mettre en doute qu'une

120 Étienne Balibar, « Le moment messianique de Marx », op. cit., p. 148.

${ }^{121}$ Karl Marx, Contribution à la critique de la philosophie du droit de Hegel. Introduction, op. cit., p. 292. 122 Ibid., p. 297.

123 G. W. F. Hegel, La philosophie de l'histoire, trad. M. Bienenstock et al., Paris, Librairie Générale Française, 2009, p. 70.

124 Voir Renaud Garcia, Le Desert de la critique. Déconstruction et politique, Paris, L'échappée, 2015. 
telle horizontalité décentralisée puisse être vraiment à la hauteur des défis à relever pour combattre le capitalisme globalisé sur fond de crise écologique planétaire ? En plus de résulter d'une méconnaissance du mode d'être de la socialité humaine, le refus de l'institutionnalisme propre à la déconstruction heurte de plein fouet les résidus de common decency encore vivante ; pour reprendre le constat d'actualité de Hegel, « la loi est, plus que tout, le Schiboleth auprès duquel se distinguent les faux frères et les faux amis du prétendu peuple ${ }^{125} \gg$.

Du point de vue sociologique qui admet, à l'instar de la Sittlichkeit hégélienne, le concept de société en tant que totalité, les utopies prônant l'horizontalité normative suscitent de sérieux doutes quant à leur viabilité. En plus de la contradiction qui consiste à vouloir l'émancipation en s'opposant à l'État en contexte de catastrophes économiques et écologiques, les tenants contemporains de l'« association des producteurs libres » présupposent une conception abstraite de l'être humain en le considérant comme naturellement enclin à la bonne entente avec autrui, avec ses associés. Il n'est pas exagéré d'admettre que la pensée critique est aujourd'hui fortement allergique à l'idée trinitaire reconnaissant comme condition de la liberté collective et individuelle la nécessité d'une libre-aliénation à un tiers symbolique. Il faut à notre avis prendre au sérieux les avertissements que l'on retrouve dans les travaux de Christopher Lasch sur le narcissisme contemporain, de Dany-Robert Dufour sur les « égo-grégaires » et de Jean-Pierre Lebrun sur les « néo-sujets » rassemblés dans des «communautés de déni »; tous ces travaux nous permettent de percevoir ce que signifie des individus de la multitude " libérés » de la médiation du tiers symbolique institué ; tous ces travaux nous présentent une subjectivité asservie au sujet automate du capital et disposée à être mobilisée dans d'éventuels délires collectifs mortifères. Il faut donc cesser de confondre l'aliénation morbide au fétichisme de la marchandise avec la libre-aliénation à la loi permettant la castration symbolique ${ }^{126}$ nécessaire à la disposition d'esprit politique.

Pour ce faire, la pensée critique doit renoncer à l'anthropocentrisme moderne qui a perdu à jamais le recours à la médiation abstraite des Lumières de la raison universelle. L'anthropocentrisme devient narcissisme lorsque les sociétés déclarent la mort de Dieu, de la raison et du sujet, et c'est pour cela qu'il est urgent de retrouver la présence d'un tiers accessible à la raison pensante comme permet de se le représenter le fait scientifiquement prouvé du phénomène de la vie sur Terre en surmontant le réductionnisme biologique et en assumant toute l'aura mystique qu'on peut ressentir, par exemple, à la naissance d'un enfant ${ }^{127}$. C'est assurément autour de la notion de vie qu'une réconciliation est possible entre les penseurs de la vie concrète qu'ont été Hegel et Marx.

125 G. W. F. Hegel, Principes de la philosophie du droit, op. cit., p. 123.

${ }^{126}$ Confusion malheureuse que l'on retrouve dans Franck Fischbach, " Présentation », dans Karl Marx, Manuscrits économico-philosophiques de 1844, op. cit., p. 38.

127 Voir Hans Jonas, Le principe responsabilité, Paris, Flammarion, 2009, p. 250 sq. 